\title{
TOPOLOGICAL TAMENESS OF MARGULIS SPACETIMES
}

\author{
SUHYOUNG CHOI AND WILLIAM GOLDMAN
}

Dedicated to the memory of Bill Thurston, our mentor

\begin{abstract}
We show that Margulis spacetimes without parabolic holonomy elements are topologically tame. A Margulis spacetime is the quotient of the 3-dimensional Minkowski space by a free proper isometric action of the free group of rank $\geq 2$. We will use our particular point of view that the Margulis spacetime is a manifold-with-boundary with an $\mathbb{R} \mathrm{P}^{3}$-structure in an essential way. The basic tools are a bordification by a closed $\mathbb{R P}^{2}$-surface with free holonomy group, and the work of Goldman, Labourie, and Margulis on geodesics in the Margulis spacetimes and 3-manifold topology.
\end{abstract}

\section{Contents}

1. Introduction

Notation and terminology 8

2. Projective geometry 9

2.1. Projective space 9

2.2. The sphere of directions 9

2.3. A matrix lemma 11

2.4. The space of properly convex sets and joins 12

3. Affine 3-dimensional Lorentzian geometry 14

3.1. The 3-dimensional Minkowski space 14

3.2. Lorentz isometries 14

3.3. Oriented Lorentzian vector spaces

Date: May 27, 2018.

1991 Mathematics Subject Classification. Primary 57M50; Secondary 53C15, 53C50, 53A20.

Key words and phrases. Lorentzian manifold, handlebody, geodesic flow, real projective structure, proper action.

The first author was supported by the National Research Foundation of Korea(NRF) grant funded by the Korea government(MEST) (No.2010-0027001). The second author gratefully acknowledges partial support from National Science Foundation grants DMS-070781 and DMS-1065965. 
3.4. Null half-planes

3.5. Affine boosts 18

3.6. Projective boosts 19

3.7. Sequences of projective boosts 22

4. Dynamics and hyperbolic geometry 24

4.1. $\mathbb{R P}^{2}$-structures on surfaces 24

4.2. Complete hyperbolic surfaces and Fuchsian groups 25

4.3. The dynamics of geodesic flows on hyperbolic surfaces 26

5. Real projective Schottky uniformizations 28

5.1. The construction of the $\Gamma$-invariant domain 28

5.2. The $\mathbb{R} P^{2}$-surface 31

6. $\Gamma$ acts properly on $\mathrm{E} \cup \tilde{\Sigma}$. 35

6.1. Diffused Margulis invariants 35

6.2. Neutralized sections 35

6.3. Lifting the neutralized section to the coverings 36

6.4. Mapping the convex core 36

6.5. The proof of proper discontinuity 37

6.6. Convergences of the axes 38

6.7. Conjugating to a standard boost form 39

6.8. Normalization 40

6.9. The convergence of convex balls 41

6.10. The balls which are bounded away from $S_{\infty}$

6.11. The balls meeting $S_{\infty}$. 43

6.12. Conclusion of the proof when attractors and repellers differ 44

6.13. Conclusion of the proof when attractors and repellers coincide 45

7. Tameness. 45

7.1. The compactification 45

7.2. The almost-crooked-plane decomposition 47

References 48

\section{InTRODUCTION}

A complete affine manifold is a quotient $M$ of an $n$-dimensional real affine space $E$ by a discrete group $\Gamma$ of affine transformations acting properly. Equivalently, $M$ is a manifold with a geodesically flat torsionfree affine connection.

Complete affine 3-manifolds with solvable fundamental groups were classified by Fried-Goldman [39], and these include all cases where $M$ is compact. In all other cases, $M$ admits a parallel Lorentzian structure, and $\pi_{1}(M) \cong \Gamma$ is a free group. The existence of these manifolds was 
first demonstrated by Margulis [53], and later clarified by Drumm [31] using crooked planes. In particular Drumm's examples are tame, that is, homeomorphic to the interiors of compact manifolds-with-boundary, which in this case are closed solid handlebodies.

Charette, Drumm, Goldman, and Labourie 31, 33, 34 made contributions to understanding such spacetimes with parallel Lorentzian structures culminating in recent work [46] and [45]. The complete classification of such spacetimes is currently going on with the work of Charette, Drumm, Goldman, Labourie, Margulis and Minsky [15], [16], [46], and [47].

The theory of compactifying open manifolds goes back to Browder, Levine and Livesay [10 and Siebenmann 60]. For 3-manifolds, Tucker [64] and Scott and Tucker [59] made an initial clarification. (See also Meyers [55]. ) As a note, we state that complete hyperbolic 3-manifolds with finitely generated fundamental groups were shown to be tame by Agol [3], Calegari, and Gabai [12]. For examples of nontame finitely generated 3-manifolds, see the examples of Scott-Tucker [59] and Canary-Minsky [13], and Example 4 of Souto [61]. Also, see Bowditch [9] for details on this very large topic. We won't elaborate on this as the methods are completely different. Our proof is closer to the proof of the tameness of geometrically finite hyperbolic 3-manifolds due to Marden [52] (see Thurston [63] and Epstein and Marden [37]. See also Bonahon [8]).

Let $V$ be a vector space over $\mathbb{R}$. The projectivization $\mathrm{P}(V)$ is defined as the quotient space

$$
V-\{O\} / \sim \text { where } \mathrm{v} \sim \mathrm{w} \text { if and only if } \mathrm{v}=s \mathrm{w} \text { for } s \in \mathbb{R}-\{0\} .
$$

Define $\mathbb{S}(V)$ as

$$
V-\{O\} / \sim \text { where } \mathrm{v} \sim \mathrm{w} \text { if and only if } \mathrm{v}=s \mathrm{w} \text { for } s>0 .
$$

A projective subspace of $\mathbf{S}^{n}$ is the image of $\mathbb{S}(W)$ of the vector subspace $W$ of $\mathbb{R}^{n+1}$ under the projection $\mathbb{R}^{n+1}-\{O\} \rightarrow \mathbb{S}\left(\mathbb{R}^{n+1}\right)=\mathbf{S}^{n}$.

Let $\mathrm{V}$ denote a 3 -dimensional real vector space with a Lorentzian inner product of signature 2,1 and a fixed orientation. The spacetime $\mathrm{E}$ is an affine space with the underlying vector space $\mathrm{V}$. A Lorentzian isometry is an automorphism of $\mathrm{E}$ preserving the Lorentzian inner product. Denote the group of orientation-preserving Lorentzian isometries by $\operatorname{Isom}^{+}(\mathrm{E}) . \mathrm{P}(\mathrm{V})$ is identical with the real projective plane $\mathbb{R} \mathrm{P}^{2}$. Here $\operatorname{PGL}(3, \mathbb{R})$ acts faithfully on $\mathbb{R P}^{2}$ as the group of collineations. Recall that we embed the hyperboloid model $\mathbb{H}^{2}$ of the hyperbolic plane to the disk that is the interior of the conic in $\mathbb{R P}^{2}$ determined by null vectors.

Define the sphere of directions $\mathbf{S}_{\infty}^{2}:=\mathbb{S}(\mathrm{V})$ double-covering $\mathbb{R P}^{2}$. Then the image $\mathbb{S}_{+}$of the space of future timelike vectors identifies 
with the hyperbolic 2-plane $\mathbb{H}^{2}$, which is basically the Beltrami-Klein model of the hyperbolic plane. Let $\mathbb{S}_{-}$denote the subspace of $\mathbb{S}$ corresponding to past timelike vectors. The group of orientation preserving linear maps of $\mathrm{V}$ is denoted by $\mathrm{SO}(2,1)$, a Lie group with two components one of which is the identity component $\mathrm{SO}(2,1)^{\circ}$. The linear group $\mathrm{SO}(2,1)^{\circ}$ acts faithfully on $\mathbb{H}^{2}=\mathbb{S}_{+}$as the orientation-preserving isometry group and $\mathbf{S O}(2,1)$ acts so on $\mathbb{S}_{+} \cup \mathbb{S}_{-}$and acts on $\mathbf{S}_{\infty}^{2}$ projectively where the action is induced from that on $\mathrm{V}$.

Since $\mathrm{SO}(2,1)$ injects to $\operatorname{PGL}(3, \mathbb{R})$ under projection $\mathrm{GL}(3, \mathbb{R}) \rightarrow$ $\operatorname{PGL}(3, \mathbb{R})$, we consider that $\mathrm{SO}(2,1)$ acts on $\mathbb{S}_{+}=\mathbb{H}^{2}$ a subset of $\mathbb{R P}^{2}$ as well in a projective manner. Additionally, $\mathrm{SO}(2,1)^{\circ}$ acts on $\mathbb{S}_{+}$ honestly as a subset of $\mathbf{S}_{\infty}^{2}$.

Denote by

$$
\operatorname{Isom}^{+}(\mathrm{E}) \stackrel{\mathbb{L}}{\rightarrow} \mathrm{SO}(2,1)
$$

obtained by taking the linear part of an affine isometry of $\mathrm{E}$. We also denote by

$$
\mathbb{L}^{\prime}: \operatorname{Isom}^{+}(\mathrm{E}) \stackrel{\mathbb{L}}{\rightarrow} \mathrm{SO}(2,1) \hookrightarrow \operatorname{PGL}(3, \mathbb{R})
$$

with the image acting on $\mathbb{R P}^{2}$. The identity component $\operatorname{Isom}_{0}^{+}(\mathrm{E})$ of Isom ${ }^{+}(\mathrm{E})$ acts on $\mathbb{S}_{+}$and $\mathbb{S}_{-}$respectively and where $\mathbb{L}\left(\operatorname{Isom}_{0}^{+}(\mathrm{E})\right) \subset$ $\mathrm{SO}(2,1)^{o}$ holds.

A linear Lorentzian isometry is hyperbolic if its eigenvalues are distinct and real. Hyperbolic isometries comprise an open subset of $\mathrm{SO}(2,1)$, and in a suitable basis represented by matrices

$$
\left[\begin{array}{ccc} 
\pm e^{l} & 0 & 0 \\
0 & 1 & 0 \\
0 & 0 & \pm e^{-l}
\end{array}\right]
$$

(where both signs agree). If all the eigenvalues are positive, then such an isometry is said to be positive hyperbolic.

Suppose that $\Gamma$ is a finitely generated orientation-preserving Lorentzian isometry group acting freely and properly on $\mathrm{E}$. We assume that $\Gamma$ is not amenable (that is, not solvable). Since $E / \Gamma$ is aspherical, the fundamental group $\Gamma$ is torsion-free by Lemma 9.4 of [49].

- $\mathbb{L} \mid \Gamma$ is faithful and $\mathbb{L}(\Gamma)$ is discrete by $\S 2.1 .2$ of Fried-Goldman 39].

- By Mess [54], $\Gamma$ is a free group of rank $\geq 2$.

Thus, $\Gamma$ injects under $\mathbb{L}$ to $\mathbb{L}(\Gamma) \subset \mathrm{SO}(2,1)$ acting properly and freely on $\mathbb{S}_{+} \cup \mathbb{S}_{-}$. We obtain an exact sequence

$$
1 \rightarrow \Gamma^{\prime} \rightarrow \Gamma \rightarrow J \rightarrow 1
$$

where $\Gamma^{\prime}$ is the subgroup of $\Gamma$ of index $\leq 2$ so that $\mathbb{L}\left(\Gamma^{\prime}\right) \subset \operatorname{SO}(2,1)^{o}$ and $J$ is either $\mathbb{Z} / 2 \mathbb{Z}$ or is the trivial group. Since $\Gamma$ acts freely on $E$, 
we obtain that $\mathbb{L}(\Gamma)$ cannot contain $-\mathrm{I}$. Hence, $\mathbb{L}^{\prime}$ is faithful. (See also $\S 3$ of Goldman-Labourie [45] for details.) Thus, we restrict ourselves to the group actions of free groups of rank $\geq 2$ in this paper. Then $\mathrm{E} / \Gamma$ is said to be a Margulis spacetime, and an element of $\Gamma$ is said to be a holonomy.

Note that $\mathbb{H}^{2} / \mathbb{L}^{\prime}(\Gamma)$ is not orientable if $\mathbb{L}(\Gamma)$ is not a subset of $\mathrm{SO}(2,1)^{o}$. However, $\mathbb{S}_{+} \cup \mathbb{S}_{-} / \mathbb{L}(\Gamma)$ is an orientable surface doublecovering $\mathbb{H}^{2} / \mathbb{L}^{\prime}(\Gamma)$. (See [16] for details.) A subgroup of $\mathrm{SO}(2,1)$ is Fuchsian if it is a discrete subgroup without torsion. A Fuchsian group $\mathbb{L}^{\prime}(\Gamma)$ is convex cocompact if one of the following holds:

- $\mathbb{H}^{2} / \mathbb{L}^{\prime}(\Gamma)$ has a compact convex core.

- $\mathbb{H}^{2} / \mathbb{L}^{\prime}(\Gamma)$ is geometrically finite without cusps

- $\mathbb{L}^{\prime}(\Gamma)$ contains no parabolic element.

In this paper, we will be concerned with the cases without parabolic elements in $\Gamma$. The following conditions are equivalent conditions for a finitely generated orientation-preserving Lorentzian isometry group $\Gamma$.

- $\mathrm{E} / \Gamma$ is a Margulis spacetime without parabolic holonomy elements.

- An index-two subgroup $\Gamma \cap \mid \operatorname{som}_{0}^{+}(\mathrm{E})$ or $\Gamma$ itself is a proper affine deformation of a convex cocompact Fuchsian group. (See [46] for definition. )

- $\Gamma$ is a nonamenable Lorentzian isometry group acting properly on $\mathrm{E}$ without parabolic holonomy.

- $\Gamma$ is a free Lorentzian isometry group of rank $\geq 2$ acting properly and freely on $\mathrm{E}$ without parabolic holonomy.

In this case, we call $\mathrm{E} / \Gamma$ a Margulis spacetime without cusp for shortness.

Notice that the definitions of hyperbolic, positive hyperbolic, and parabolic for elements of $\Gamma$ are the same as ones for $\mathbb{L}^{\prime}(\Gamma)$ as a group of isometries of $\mathbb{S}_{+}$. (If a hyperbolic element in $\mathrm{SO}(2,1)$ has a negative eigenvalue, then the eigenvalues should be $-\lambda,-1 / \lambda, 1$, where $\lambda>0$.) It also follows from above that elements of $\Gamma$ are either hyperbolic or parabolic or identity. (See [46]).

An $\mathbb{R P}^{n}$-structure on a manifold is given by an atlas of charts to $\mathbb{R P}^{n}$ with projective transition maps. Such geometric structures were first considered by Kuiper, Benzécri, Vinberg, Koszul and others in the 1950s and 1960s. Further developments can be followed in Goldman [44, Choi and Goldman [24], Benoist [6], and Cooper, Long, and Thistlethwaite [26] and [27] and many others.

Theorem 1.1. Let $\Gamma$ be a free orientation-preserving Lorentzian isometry group of rank $\geq 2$ acting on on $\mathbb{S}_{+} \cup \mathbb{S}_{-}$properly and freely but 
without parabolic. Then $\Gamma$ acts on an open domain $\mathcal{D} \subset \mathbf{S}_{\infty}^{2}$ such that $\mathcal{D} / \Gamma$ is a closed surface $\Sigma$. Such a domain is unique up to the antipodal map $\mathscr{A}$.

These surfaces have the $\mathbb{R P}^{2}$-structures on closed surfaces of genus $g, g \geq 2$, discovered by Goldman [42] in the late 1970s. Although their developing maps from the universal cover $\tilde{\Sigma}$ of some such surface $\Sigma$ are not covering-spaces onto their images in $\mathbb{R P}^{2}$, when lifted to the double-covering space $\mathbf{S}_{\infty}^{2}$, the developing maps (remarkably) are covering-spaces onto open domains. The surface is a quotient of a domain in $\mathbf{S}_{\infty}^{2}$ by a group of projective automorphisms, an $\mathbb{R P}^{2}$-analog of the standard Schottky uniformization of a Riemann surface as a $\mathbb{C P}^{1}$-manifold as observed by Goldman.

An $n$-dimensional open manifold is said to be tame if it is homeomorphic to the interior of a compact $n$-manifold with boundary. A handlebody is a 3-dimensional manifold obtained from a 3 -ball $B^{3}$ by attaching 1-handles to 3-dimensional balls. The topology of handlebodies and related objects form a central topic in the 3-dimensional manifold topology. See Hempel [49] for a thorough survey of this field of mathematics developed in the 20th century.

Theorem 1.2. Let $M$ be the Margulis spacetime $\mathrm{E} / \Gamma$ without cusp. Then $M$ is homeomorphic to the interior of an orientable handlebody.

The proof is given in $\$ 7$ by compactifying the Margulis spacetime into a compact $\mathbb{R} P^{3}$-manifold with $\mathbb{R P}^{2}$-surface boundary. We remark that Frances [38] earlier produced conformal compactifications of Margulis spacetimes, which are not manifolds as opposed to our projective compactifications. We are currently trying to generalize these techniques to Lorentz manifolds with parabolics (see [25]) and to higher dimensional geometric structures and so on.

A complete geodesic on a quotient space of a hyperbolic space or a Lorentzian space is nonwandering if it is bounded in both directions, or equivalently, the closure of the forward part is compact and so is that of the backward part, or the $\alpha$-limit and the $\omega$-limit are both nonempty and compact. This is the same as the term "recurrent" in [45] and [18], which does not agree with the common usage in the dynamical system theory whereas the recurrence set they discuss is the same as the nonwandering set as in Eberlein [35] and Katok and Hasselblatt [50]. Both endpoints of the geodesics lie in the limit sets and they comprise the nonwandering set by Corollary 3.8 in [35].

In \$2, we review facts on hyperbolic surfaces, Fuchsian group actions, $\mathbb{R P}^{2}$-structures and geometry that we need. We compactify $\mathrm{E}$ by a real projective 2-sphere $\mathbf{S}_{\infty}^{2}$ that is just the space of directions in $\mathbf{E}$. 
In $\$ 3$, we review facts about the Lorentzian space $\mathrm{E}$ and affine boosts. The affine boosts will be extended to projective boosts in $\mathbf{S}^{3}$.

In $\$ 4$, we review $\mathbb{R P}^{2}$-structures on surfaces and hyperbolic surfaces. We also discuss the dynamics of the geodesic flows on the unit tangent bundles of hyperbolic surfaces.

In $\$ 5$, we prove Theorem 1.1 . We find an open domain $\tilde{\Sigma}$ in the boundary $\mathbf{S}_{\infty}^{2}$ of $\mathrm{E}$ where $\Gamma$ acts properly and freely. $\tilde{\Sigma} / \Gamma$ is shown to be a closed $\mathbb{R P}^{2}$-surface $\Sigma$. Such a surface was constructed by Goldman 42]; however, we realize it as the quotient of an open domain in the sphere $\mathbf{S}_{\infty}^{2}$, which is a union of two disks and infinitely many strips joining the two. Here our choice of $\tilde{\Sigma}$ depends on the orientation.

In \$6, we recall the work of Goldman, Labourie, and Margulis [46] on nonwandering geodesics on the Margulis spacetime. Their work in fact shows that all nonwandering spacelike geodesics in a Margulis spacetime are in a uniformly bounded part.

We fix an orientation on $\mathrm{E}$ and assume that the Margulis invariants are all positive. Our choice of $\tilde{\Sigma}$ above depends on the orientations and the signs of Margulis invariants. (See the statements after Theorem 7.2.) Next, we prove the proper discontinuity of the action of the group $\Gamma$ on $E \cup \tilde{\Sigma}$. We first show that for a fixed Lorentzian isometry $g$, we can put every compact properly convex subset of $E \cup \tilde{\Sigma}$ into an $\epsilon$-neighborhood of the "attracting" segment of $g$ in $\mathbf{S}_{\infty}^{2}$ by $g^{i}$ for sufficiently large $i$. (This is the key model of our proof.)

We next show that for any compact set $K \subset \mathrm{E} \cup \tilde{\Sigma}$, we have only finitely many $g \in \Gamma$ where $g(K) \cap K \neq \emptyset$. Suppose not. We find a sequence $\left\{g_{i}\right\}$ with $g_{i}(K) \cap K \neq \emptyset$. Using [46], we can find an infinite sequence $\left\{g_{i}\right\}$ that behaves almost like $\left\{g^{i}\right\}$ in the dynamical sense as $i \rightarrow \infty$ up to small changes of stable and unstable planes. In other words, we can find a uniformly bounded sequence of coordinates to normalize $g_{i}$ into fixed forms and these coordinates are uniformly convergent.

In $\$ 7$, we prove the tameness of $\mathrm{E} / \Gamma$, i.e., Theorem 1.2 , using the classical 3-manifold topology as developed by Dehn and others. We conclude with the compactification Theorem 7.2 , proving Theorem 1.2 .

There is another independent solution to this question by Danciger, Guéritaud, and Kassel [28]. Their approach uses the deformation of constant curvature Lorentzian manifolds and yields other results such as the deformability of Margulis spacetimes to anti-de Sitter spaces. Their method is to identify $\mathbb{R}^{3}$ as the Lie algebra $\operatorname{sl}(2, \mathbb{R})$ with the adjoint action by $\mathrm{SL}(2, \mathbb{R})$. Then they show the existence of foliation of $M$ by complete lines fibering over the corresponding hyperbolic surface. Compared to their approach, our approach mostly concentrates 
on $\mathbb{R P}^{n}$-structures and the compactification of the spacetime as an $\mathbb{R} P^{3}$-manifold.

Danciger, Guéritaud, and Kassel [29] also announced in September 2013 in ICERM the solution to the crooked plane conjecture of DrummGoldman 32] that the Margulis spacetimes without cusp decomposes into 3-balls by unions of mutually disjoint crooked planes. This implies the tameness also. With Theorem 1.1, we can obtain compactifications, i.e., Theorem 1.2 also. For rank two cases, the conjecture was verified by Charette-Drumm-Goldman [17]. Related to this conjecture, we have Proposition 7.3 .

This work began from the question of Goldman on the $\mathbb{R P}^{2}$-bordification of the Margulis spacetime during the Newton Institute Workshop "Representations of Surface Groups and Higgs Bundles" held in Oxford, March 14-18, 2011. The authors thank the Newton Institute and the Institut Henri Poincaré where parts of this work were carried out. Finally, we thank Bill Thurston without whose teaching we could not have accomplished many of the things in this paper.

\section{NOTATION AND TERMINOLOGY}

Let $V$ denotes a (real) vector space. We denote the associated projective space, comprising 1-dimensional linear subspaces of $V$ by $\mathrm{P}(V)$. Projective $n$-space $\mathbb{R} \mathrm{P}^{n}:=\mathrm{P}\left(\mathbb{R}^{n+1}\right)$ also identifies with the quotient of $\mathbb{R}^{n+1} \backslash\{0\}$ by the multiplicative group $\mathbb{R}^{*}$ of nonzero real scalars. The sphere of directions $\mathbf{S}^{n}$ consists of the quotient of $\mathbb{R}^{n+1}-\{0\}$ by the subgroup $\mathbb{R}^{+}$of positive real scalars. If $v \in \mathbb{R}^{n+1}$ and $v \neq 0$, then denote its equivalence class in $\mathbb{R P}^{n}$ by $[v]=\left[v^{0}: v^{1}: \cdots: v^{n}\right]$ and its equivalence class in $\mathbf{S}^{n}$ by $((v))=\left(\left(v^{0}: v^{1}: \cdots: v^{n}\right)\right)$. The map

$$
\begin{aligned}
\mathrm{S}^{n} & \longrightarrow \mathbb{R} \mathrm{P}^{n} \\
((v)) & \longmapsto[v]
\end{aligned}
$$

is a double covering. The coordinates $v^{0}, \ldots v^{n}$ are the homogeneous coordinates of the corresponding points $[v] \in \mathbb{R} \mathrm{P}^{n}$ and $((v)) \in \mathbf{S}^{n}$.

The sign of a real number $x$ is defined as:

$$
\operatorname{sgn}(x):= \begin{cases}+1 & \text { if } x>0 \\ 0 & \text { if } x=0 \\ -1 & \text { if } x<0\end{cases}
$$

If $G$ is a group acting on a space $X$, and $S \subset X$ is a subset, then denote its stabilizer by:

$$
\operatorname{Stab}(S):=\{g \in G \mid g(S)=S\} .
$$




\section{Projective Geometry}

2.1. Projective space. The projective space $\mathbb{R} \mathrm{P}^{n}$ is given by $\mathrm{P}\left(\mathbb{R}^{n+1}\right)$. We have a homomorphism from the general linear group

$$
\operatorname{GL}(n+1, \mathbb{R}) \rightarrow \operatorname{PGL}(n+1, \mathbb{R}):=\operatorname{GL}(n+1, \mathbb{R}) / \sim
$$

where two linear maps $L_{1}$ and $L_{2}$ are equivalent if $L_{1}=s L_{2}$ for $s \in$ $\mathbb{R}-\{0\}$. A map of an open subset of $\mathbb{R} \mathrm{P}^{n}$ to another one of $\mathbb{R P} \mathrm{P}^{n}$ is projective if it is a restriction of an element of $\operatorname{PGL}(n+1, \mathbb{R})$.

More generally, a nonzero linear map $L: \mathbb{R}^{n+1} \rightarrow \mathbb{R}^{n+1}$ becomes a projective endomorphism

$\hat{L}: \mathrm{P}\left(\mathbb{R}^{n+1}\right)-\mathrm{P}(N) \rightarrow \mathrm{P}\left(\mathbb{R}^{n+1}\right)$ defined by $\hat{L}([\vec{v}])=[L(\vec{v})], \vec{v} \in \mathbb{R}^{n+1}$,

where $N$ is the kernel of $L$ and $\mathrm{P}(N)$ is the image of $N-\{O\}$ in $\mathrm{P}\left(\mathbb{R}^{n+1}\right)$. (See $\S 5.1$ of Benzécri [7].) The space of projective endomorphisms equals the projectivization $\mathrm{P}\left(\mathrm{Mat}_{n+1}(\mathbb{R})\right)$ of the linear space $\mathrm{Mat}_{n+1}(\mathbb{R})$ of all linear maps $\mathbb{R}^{n+1} \rightarrow \mathbb{R}^{n+1}$. The space $\mathrm{P}\left(\operatorname{Mat}_{n+1}(\mathbb{R})\right)$ forms a compactification of the group $\operatorname{PGL}(n+1, \mathbb{R})$ as observed first by Kuiper [51] and developed by Benzécri [7].

The projective geometry is given by a pair $\left(\mathbb{R P}^{n}, \operatorname{PGL}(n+1, \mathbb{R})\right)$. An $\mathbb{R P}^{n}$-structure on a manifold $M$ is given by a maximal atlas of charts to $\mathbb{R P}^{n}$ with projective transition maps. The manifold $M$ with an $\mathbb{R P}^{n}$-structure is said to be an $\mathbb{R P}^{n}$-manifold. A projective map for two $\mathbb{R P}^{n}$-manifolds $M$ and $N$ is a map $f: M \rightarrow N$ so that $\phi \circ f \circ \psi^{-1}$ is projective whenever it is defined where $\phi$ is a chart for $N$ and $\psi^{-1}$ is a local inverse of a chart for $M$.

An $\mathbb{R P}^{n}$-structure on a manifold $M$ gives us an immersion dev $: \tilde{M} \rightarrow$ $\mathbb{R} \mathrm{P}^{n}$ that is equivariant with respect to a homomorphism

$$
\begin{aligned}
& h: \pi_{1}(M) \rightarrow \operatorname{PGL}(n+1, \mathbb{R}): \text { that is, } \\
& \operatorname{dev} \circ \gamma=h(\gamma) \circ \operatorname{dev} \text { for every } \gamma \in \Gamma
\end{aligned}
$$

where $\tilde{M}$ is a regular cover of $M$ and $\Gamma$ is the deck transformation group of $\tilde{M} \rightarrow M$. Here, dev is called a developing map and $h$ is called the holonomy homomorphism. (dev, $h$ ) is only determined up to an action of $\operatorname{PGL}(n+1, \mathbb{R})$ where

$$
g(\operatorname{dev}, h(\cdot))=\left(g \circ \operatorname{dev}, g h(\cdot) g^{-1}\right) \text { for } g \in \operatorname{PGL}(n+1, \mathbb{R}) .
$$

Conversely, such a pair (dev, $h$ ) will give us an $\mathbb{R P}^{n}$-structure since dev gives us charts that have projective transition maps. (See [20] for details.)

2.2. The sphere of directions. We can identify the sphere $\mathbf{S}^{n}$ with $\mathbb{S}\left(\mathbb{R}^{n+1}\right)$. There exists a quotient map $q_{1}: \mathbf{S}^{n} \rightarrow \mathbb{R P}^{n}$ defined by sending a unit vector to its equivalence class. This is a double-covering 
map, and it induces an $\mathbb{R P}^{n}$-structure on $\mathbf{S}^{n}$. The group $\operatorname{Aut}\left(\mathbf{S}^{n}\right)$ of projective automorphisms is isomorphic to the group $\mathrm{SL}_{ \pm}(n+1, \mathbb{R})$ of linear maps of determinant equal to \pm 1 with the quotient homomorphism $\mathrm{SL}_{ \pm}(n+1, \mathbb{R}) \rightarrow \operatorname{PGL}(n+1, \mathbb{R})$ with the kernel $\pm \mathrm{I}$. It will be convenient to think of the elements of $\operatorname{Aut}\left(\mathbf{S}^{n}\right)$ as matrices in $\mathrm{SL}_{ \pm}(n+1, \mathbb{R})$.

This space is again an open dense subspace of $\mathbb{S}\left(\operatorname{Mat}_{n}(\mathbb{R})\right)$. We call each element of $\mathbb{S}\left(\operatorname{Mat}_{n}(\mathbb{R})\right)$ a projective endomorphism of $\mathbf{S}^{n}$. Each element corresponds to a map

$$
\hat{L}: \mathbb{S}\left(\mathbb{R}^{n+1}\right)-\mathbb{S}(N) \rightarrow \mathbb{S}\left(\mathbb{R}^{n+1}\right) \text { given by } \hat{L}(((\vec{v})))=((L(\vec{v}))), \vec{v} \in \mathbb{R}^{n+1}
$$

where $N$ is the kernel of a linear map $L$ in $\operatorname{Mat}_{n}(\mathbb{R})$.

For an open domain $\mathcal{D}$ in $\mathbf{S}^{n}$ and a discrete group $\Gamma$ in $\operatorname{Aut}\left(\mathbf{S}^{n}\right)$ acting on it properly and freely, $\mathcal{D} / \Gamma$ has an $\mathbb{R P}^{n}$-structure since $q_{1} \mid \mathcal{D}$ gives an immersion to $\mathbb{R} \mathrm{P}^{n}$ and the homomorphism

$$
\Gamma \subset \operatorname{Aut}\left(\mathbf{S}^{n}\right) \rightarrow \operatorname{PGL}(n+1, \mathbb{R})
$$

gives the associated holonomy homomorphism.

In this paper, we will study objects on $\mathbf{S}^{3}=\mathbb{S}\left(\mathbb{R} \oplus \mathbb{R}^{3}\right)$.

The antipodal map

$$
\begin{aligned}
& \mathbf{S}^{n} \stackrel{\mathscr{A}}{\longrightarrow} \mathbf{S}^{n} \\
& ((\mathrm{v})) \longmapsto((-\mathrm{v}))
\end{aligned}
$$

generates the deck transformation group $\mathbf{S}^{n} \rightarrow \mathbb{R} \mathrm{P}^{n}$. Given a subset or a point $K$ of $\mathbf{S}^{3}$, we denote by $K_{-}$the set of antipodal points of points of $K$ or the antipodal point respectively.

Given a subset $A$ of $\mathbf{S}^{n}, \operatorname{span}(A)$ is the unique minimal projective subspace $S$ of $\mathbf{S}^{n}$ containing $A$.

2.2.1. Elliptic geometry. A subspace of $\mathbf{S}^{3}$ is a subset defined by a system of linear equations in $\mathbb{R}^{4}$. A line is a 1-dimensional subspace. A singleton is not a subspace. A projective geodesic is an arc in a line. We will be using the standard Riemannian metric $\mathbf{d}$ on $\mathbf{S}^{3}$. Notice that the geodesic structure of $\mathbf{S}^{3}$ is the same as the projective one. Thus, we will use the same term "geodesics" for both types of geodesics. Geodesics and projective subspaces are all subsets of lines or subspaces in $\mathbf{S}^{3}$. A projective automorphism $g$ sends these to themselves, while $g$ does not preserve any metric. A pair of antipodal points on $\mathbf{S}^{3}$ is the equivalence class $((v))$ of a nonzero vector $v$ and one $((-v))$ of $v_{-}$. A segment connecting two antipodal points is precisely a segment of $\mathbf{d}$-length $\pi$. (For these topics, see $\S 34$ of Eisenhart [36].)

Here, we denote by $\overline{p q p_{-}}$the unique closed segment in $\mathbf{S}^{3}$ with endpoints $p$ and $p_{-}$passing through $q$ not equal to $p$ or $p_{-}$. By $\overline{p q}$, we 
denote the unique closed segment in $\mathbf{S}^{3}$ with endpoints $p$ and $q$ not containing $p_{-}, q_{-}$provided $p \neq q$ and $p \neq q_{-}$. The notation $\overline{p q}$ or ${\overline{p q p_{-}}}^{\circ}$ indicates the interior of the segment.

A convex subset of $\mathbf{S}^{3}$ is a subset where every pair of points can be connected by a segment of $\mathbf{d}$-length $\leq \pi$. A subset $A$ of $\mathbf{S}^{3}$ is properly convex if $A$ is a precompact convex subset of some open hemisphere. A compact convex set is properly convex if and only if there are no antipodal pair of points. These notions are projectively invariant. (See [23] for details.)

2.2.2. Bi-Lipschitz maps. A bi-Lipschitz map of $\mathbf{S}^{3}$ is a homeomorphism $\mathbf{S}^{3} \stackrel{f}{\rightarrow} \mathbf{S}^{3}$ so that

$$
C^{-1} \mathbf{d}(x, y) \leq \mathbf{d}(f(x), f(y)) \leq C \mathbf{d}(x, y) \text { for } x, y \in \mathbf{S}^{3}
$$

where $C>0$ is independent of $x, y$. Elements of Aut $\left(\mathbf{S}^{3}\right)$ including ones extending the Lorentzian isometries are bi-Lipschitz maps of $\mathbf{S}^{3}$.

Finally, for the purpose of drawing figures only, we map $E$ to the unit 3-ball in $\mathbb{R}^{3}$ by the normalization map

$$
((x: y: z: 1)) \rightarrow \frac{(x, y, z)}{\sqrt{x^{2}+y^{2}+z^{2}+1}} .
$$

This will identify $\mathbf{S}_{\infty}^{2}$ with the sphere of radius 1 in $\mathbb{R}^{3}$ and as a codimension-one projective subspace of $\mathbf{S}^{3}$, represented stereographically.

\subsection{A matrix lemma. We denote}

$$
\begin{aligned}
& \mathrm{e}_{1}=\left(\left(\vec{e}_{1}\right)\right), \mathrm{e}_{2}=\left(\left(\vec{e}_{2}\right)\right), \mathrm{e}_{3}=\left(\left(\vec{e}_{3}\right)\right), \mathrm{e}_{4}=\left(\left(\vec{e}_{4}\right)\right), \\
& \mathrm{e}_{1-}=\left(\left(-\vec{e}_{1}\right)\right), \mathrm{e}_{2-}=\left(\left(-\vec{e}_{2}\right)\right), \mathrm{e}_{3-}=\left(\left(-\vec{e}_{3}\right)\right), \mathrm{e}_{4-}=\left(\left(-\vec{e}_{4}\right)\right) .
\end{aligned}
$$

There exists a standard metric on $\mathbb{R}^{4}$ where these vectors form an orthonormal basis. We will use the norm of this metric to be called the Euclidean norm. The norm of a matrix $A$ is $\max \left\{\left|a_{i j}\right|\right\}_{i=1, \ldots, 4, j=1, \ldots, 4}$ for entries $a_{i j}$ of $A$.

Lemma 2.1 (Uniform convergence). Let $\mathbf{v}_{i}^{j}$ for $j=1,2,3,4$ be a sequence of points of $\mathbf{S}^{3}$. Suppose that $\mathbf{v}_{i}^{j} \rightarrow \mathrm{v}_{\infty}^{j}$ for each $j$ and mutually distinct points $\mathrm{v}_{\infty}^{1}, \ldots, \mathrm{v}_{\infty}^{4}$ formed by independent vectors in $\mathbb{R}^{4}$. Then there exists an integer $I_{0}$ and a sequence $h_{i}$ for $i>I_{0}$ of elements of $\operatorname{Aut}\left(\mathbf{S}^{3}\right)$ so that

- $h_{i}\left(\mathrm{v}_{i}^{j}\right)=\mathrm{e}_{j}$,

- $\left\{h_{i}\right\} \rightarrow h_{\infty}$ and $\left\{h_{i}^{-1}\right\} \rightarrow h_{\infty}^{-1}$ uniformly under the $C^{s}$-topology for every $s \geq 0$. 
Proof. Let $\vec{v}_{i}^{j}$ represent the unit Euclidean norm vector corresponding to $\mathrm{v}_{i}^{j}$. We find $\hat{h}_{i} \in \operatorname{Aut}\left(\mathbf{S}^{3}\right)$ so that $\hat{h}_{i}\left(\mathrm{e}_{j}\right)=\mathrm{v}_{i}^{j}$ for each $i$. We set $M_{i}\left(\vec{e}_{i}\right)=\vec{v}_{i}^{j}$; that is, the column vectors of $M_{i}$ equals $\vec{v}_{i}^{1}, \vec{v}_{i}^{2}, \vec{v}_{i}^{3}, \vec{v}_{i}^{4}$. Then clearly, the sequence $\left\{M_{i}\right\}$ converges to a $4 \times 4$-matrix $M_{\infty}$ of nonzero determinant. Therefore, there exists an integer $I_{0}$ where the sequence $\left\{M_{i}^{-1}\right\}$ for $i>I_{0}$ of inverse matrices converges to $M_{\infty}^{-1}$.

We let $h_{i}$ be the projective automorphism in $\operatorname{Aut}\left(\mathbf{S}^{3}\right)$ induced by $M_{i}^{-1}$, let $h_{\infty}$ be the one corresponding to $M_{\infty}^{-1}$, and this satisfies the properties we need.

2.4. The space of properly convex sets and joins. Let $\mathbf{d}-\operatorname{diam}(A)$ denote the diameter of the set; that is, the supremum of the set of distances between every pair of points of a subset $A$ of $\mathbf{S}^{n}$. Given a pair of points or subsets $A$ and $B$ in $\mathbf{S}^{n}$, we define the infimum distance

$$
\mathbf{d}(A, B)=\inf \{\mathbf{d}(x, y) \mid x \in A, y \in B\} .
$$

If $\mathbf{d}(A, B)>\epsilon$ for $\epsilon>0$, then we say that $A$ is bounded away from $B$ by $\epsilon$.

An $\epsilon$-neighborhood $N_{\epsilon}(A)$ for a number $\epsilon>0$ of a subset or a point $A$ of $\mathbf{S}^{3}$ is the set of points of $\mathbf{d}$-distances less than $\epsilon$ from some points of $A$. We define the geometric Hausdorff distance $\mathbf{d}^{H}(A, B)$ between two compact subsets $A$ and $B$ of $\mathbf{S}^{3}$ to be

$$
\inf \left\{\epsilon>0 \mid B \subset N_{\epsilon}(A) \text { and } A \subset N_{\epsilon}(B)\right\} .
$$

A sequence of compact sets $\left\{K_{n}\right\}$ in $\mathbf{S}^{3}$ converges (or converges geometrically) to a compact subset $K$ if $\mathbf{d}^{H}\left(K_{n}, K\right) \rightarrow 0$. In other words, for every $\epsilon>0$, there exists $N$ so that

$$
K \subset N_{\epsilon}\left(K_{n}\right) \text { and } K_{n} \subset N_{\epsilon}(K) \text { for } n>N .
$$

We will simply write $\left\{K_{n}\right\} \rightarrow K$.

The following are commonly known facts with elementary proofs (see pages 280-281 of Munkres [56]).

- The space of compact subsets of a compact Hausdorff space with this metric is compact Hausdorff also and hence every sequence has a convergent subsequence.

- given a convergent sequence $\left\{K_{n}\right\}$ of compact subsets of $\mathbf{S}^{3}$, if a sequence $\left\{J_{n}\right\}$ is such that $J_{n} \subset K_{n}$, then any geometric limit of a subsequence of $\left\{J_{n}\right\}$ is a subset of the geometric limit of $\left\{K_{n}\right\}$.

- given sequences $\left\{K_{n}\right\}$ and $\left\{\hat{K}_{n}\right\}$ of compact subsets of $\mathbf{S}^{3}$ converging to $K_{\infty}$, if a sequence $\left\{J_{n}\right\}$ is such that $K_{n} \subset J_{n} \subset \hat{K}_{n}$ for every $n$, then $\left\{J_{n}\right\} \rightarrow K_{\infty}$. 
- If we have $\left\{K_{n}^{j}\right\} \rightarrow K^{j}$ as $n \rightarrow \infty$ for each $j=1, \ldots, m_{0}$, then

$$
\bigcup_{j=1}^{m_{0}} K_{n}^{j} \rightarrow \bigcup_{j=1}^{m_{0}} K^{j}
$$

holds as $n \rightarrow \infty$.

Note that

$$
\mathbf{d}(A, B) \leq \mathbf{d}^{H}(A, B)
$$

2.4.1. Joins. Suppose that two compact subsets $A$ and $B$ of $\mathbf{S}^{3}$ have no pairs of points $x \in A, y \in B$ so that $x$ and $y$ are antipodal, that is, $A \cap B_{-}=\emptyset$. Then the join $\operatorname{Join}(A, B)$ is the union of all segments of d-lengths $<\pi$ with single endpoints in $A$ and the other ones in $B$. In set theoretic terms, we have

$$
\operatorname{Join}(A, B)=\left\{\left(\left(c_{1} \mathbf{a}+c_{2} \mathbf{b}\right)\right) \mid((\mathbf{a})) \in A,((\mathbf{b})) \in B, c_{1}, c_{2} \geq 0\right\} .
$$

If $A$ and $B$ are convex and compact and $A \cup B$ has no pair of antipodal points, i.e., $A$ and $B$ are convex and compact and $A \cap B_{-}=\emptyset$, then Join $(A, B)$ is defined and also convex. (See Proposition 2.4 of [23].) For any projective automorphism $g \in \operatorname{Aut}\left(\mathbf{S}^{3}\right)$,

$$
g(\operatorname{Join}(A, B))=\operatorname{Join}(g(A), g(B)) .
$$

If $A$ and $B$ are compact and have no antipodal pair of a point of $A$ and a point $B$, then $\operatorname{Join}(A, B)$ is well-defined and is also compact as we can easily show that $\operatorname{Join}(A, B)$ is closed.

For example, when $A$ is a great circle in $\mathbf{S}^{3}$ and $b \notin A$, every segment from $b$ to $A$ has d-length $<\pi-\epsilon$ for a fixed $\epsilon>0$. In this case, Join $(A, B)$ is a 2-dimensional hemisphere, a disk in a hyperspace with geodesic boundary in $\mathbf{S}^{3}$, a convex set.

Lemma 2.2. Let $A_{n}$ and $B_{n}$ be a sequence of properly convex compact subsets of $\mathbf{S}^{3}$ geometrically converging to properly convex compact sets $A$ and $B$ respectively. Suppose that $A \cap B_{-}=\emptyset$. Then $J_{n}=\operatorname{Join}\left(A_{n}, B_{n}\right)$ is defined for $n>I_{0}$ for sufficiently large $I_{0}$ and the sequence $\left\{J_{n}\right\}_{n>I_{0}}$ converges to the join $J$ of $A$ and $B$.

Proof. Choose $\epsilon>0$ so that $N_{\epsilon}(A)$ and $N_{\epsilon}(B)$ are properly convex and $N_{\epsilon}(A) \cap\left(N_{\epsilon}(B)\right)_{-}=\emptyset$. Choose $I_{0}$ so that $A_{n} \subset N_{\epsilon}(A), B_{n} \subset N_{\epsilon}(B)$ for $n>I_{0}$. Clearly, $A_{n}$ and $B_{n}$ have no antipodal pair of points for $n>I_{0}$. One can parameterize each geodesic segment connecting a point of $A_{n}$ to that of $B_{n}$ by arclength in $\mathbf{d}$. From this, the lemma follows. 


\section{Affine 3-Dimensional Lorentzian GeOMEtRy}

3.1. The 3-dimensional Minkowski space. The Lorentzian space $E$ is the affine space $\mathbb{R}^{3}$ equipped with a nondegenerate bilinear form - of signature $1,1,-1$. If we choose the origin in $\mathrm{E}$, we obtain the Lorentzian vector space $\mathrm{V}$.

One identifies $\mathbb{R}^{3}$ with $E$ and the complement of a subspace of codimension one in $\mathbb{R P} P^{3}$, so called the complete affine subspace. $\mathbb{R}^{3}=E$ lifts to a subspace of $\mathbf{S}^{3}$ double-covering $\mathbb{R} \mathrm{P}^{3}$. The closure of the lifted $\mathrm{E}$ is a standard 3-dimensional hemisphere $\mathscr{H}$, and $\mathrm{E}$ identifies with the open hemisphere $\mathscr{H}^{o}$. The boundary $\mathbf{S}_{\infty}^{2}$ of $\mathbf{E}$ is the subspace of codimension one identifiable with the 2 -sphere of directions in $\mathbb{R}^{3}$.

The sphere $\mathbf{S}_{\infty}^{2}$ corresponds to the hyperplane given by $t=0$ and the origin of $\mathrm{E}$ is given by $((0: 0: 0: 1))$, denoted by $O$. For this system of coordinates, the point $(x, y, z) \in \mathrm{E}$ is given coordinates $((x: y: z: 1))$.

$\mathbf{S}_{\infty}^{2}$ also has a homogeneous coordinate system $((x: y: z))$ assigned to a vector $p$ in $\mathbf{S}_{\infty}^{2}$ of unit length if $\frac{(x, y, z)}{\|(x, y, z)\|}=p$. In the larger coordinate system $((x: y: z))$ is identical with $((x: y: z: 0))$.

We will also be using a fixed Euclidean metric $d_{E}$ on $\mathrm{E}$ compatible with the affine coordinate system. It is of great importance that projective geodesics, spherical geodesics, and Euclidean geodesics on $\mathrm{E}$ are the same ones up to parametrizations.

To summarize, the subsets of $\mathbf{S}^{3}$ identified are:

$$
\begin{aligned}
\mathbf{S}^{3} & =\{((x: y: z: t)) \mid x, y, z, t \in \mathbb{R},(x, y, z, t) \neq(0,0,0,0)\}, \\
\mathscr{H} & =\{((x: y: z: t)) \mid x, y, z, t \in \mathbb{R}, t \geq 0,(x, y, z, t) \neq(0,0,0,0)\}, \\
\mathbb{R}^{3} & =\mathrm{E}=\mathscr{H}^{o}=\{((x: y: z: t)) \mid x, y, z, t \in R, t>0\}, \\
& =\{((x: y: z: 1)) \mid x, y, z \in \mathbb{R}\}, \\
\mathbf{S}_{\infty}^{2} & =\{((x: y: z: 0)) \mid x, y, z \in \mathbb{R},(x, y, z, 0) \neq(0,0,0,0)\} .
\end{aligned}
$$

3.2. Lorentz isometries. An orientation-preserving Lorentz isometry is represented as an affine transformation

$$
\begin{aligned}
& \mathrm{E} \stackrel{\gamma}{\rightarrow} \mathrm{E} \\
& \mathbf{x} \mapsto A \mathbf{x}+\mathbf{b}
\end{aligned}
$$

for $\mathrm{x} \in \mathrm{E}$.

Such an element can be represented by a matrix

$$
\left(\begin{array}{cc}
A & \mathbf{b} \\
0 & 1
\end{array}\right) \in \mathrm{GL}(4, \mathbb{R})
$$


with a nonsingular $3 \times 3$-matrix $A$ in $\mathrm{SO}(2,1)$ and $\mathbf{b} \in \mathbb{R}^{3}$.

The group Isom $^{+}(\mathrm{E})$ of orientation-preserving Lorentzian transformations of $E$ is a subgroup of the group $\mathrm{Aff}^{+}(\mathrm{E})$ of orientation-preserving affine transformations of $\mathrm{E}$. $\mathrm{Aff}^{+}(\mathrm{E})$ identifies with the subgroup of Aut $\left(\mathbf{S}^{3}\right)$ of orientation-preserving elements acting on $\mathbb{R}^{3}=\mathrm{E}$, preserving $\mathbf{S}_{\infty}^{2}$, since any affine transformation of $\mathbb{R}^{3}$ extends to a projective automorphism of $\mathbb{R} P^{3}$ and hence to an automorphism of $\mathbf{S}^{3}$ analytically. We obtain embeddings

$$
\operatorname{Isom}^{+}(\mathrm{E}) \hookrightarrow \mathrm{Aff}^{+}(\mathrm{E}) \hookrightarrow \operatorname{Aut}\left(\mathrm{S}^{3}\right) .
$$

The elements of respective groups are called projective automorphisms of Lorentzian type and ones of affine type or just Lorentzian isometries or affine transformations.

3.3. Oriented Lorentzian vector spaces. Let $(\mathrm{V}, \cdot$, Det $)$ denote a three-dimensional oriented Lorentzian $\mathbb{R}$-vector space. That is, $\mathrm{V} \cong \mathbb{R}^{3}$ and is given a symmetric bilinear form

$$
\mathrm{V} \times \mathrm{V} \rightarrow \mathbb{R} \text { given by }(\mathrm{v}, \mathrm{u}) \mapsto \mathrm{v} \cdot \mathrm{u}
$$

of index 1, and a nondegenerate alternating trilinear form

$$
\begin{aligned}
\mathrm{V} \times \mathrm{V} \times \mathrm{V} & \longrightarrow \mathbb{R} \\
(\mathrm{v}, \mathrm{u}, \mathrm{w}) & \longmapsto \operatorname{Det}(\mathrm{v}, \mathrm{u}, \mathrm{w}) .
\end{aligned}
$$

The automorphism group of $(\mathrm{V}, \cdot$, Det $)$ is the special orthogonal group $\mathrm{SO}(2,1)$.

The Lorentzian structure divides $\mathbf{S}_{\infty}^{2}$ into three open domains $\mathbb{S}_{+}, \mathbb{S}_{0}$, and $\mathbb{S}_{-}$, which are separated by two conics $\partial \mathbb{S}_{+}$and $\partial \mathbb{S}_{-}$corresponding to the nullcone in $\mathbf{V}$. Let $\mathbb{S}_{+} \subset \mathbb{S}$ denote the set of future timelike directions; its boundary $\partial \mathbb{S}_{+}$consists of future null directions. Similarly, let $\mathbb{S}_{-} \subset \mathbb{S}$ denote the set of past timelike directions with boundary $\partial \mathbb{S}_{-}$consisting of past null directions. Both $\mathbb{S}_{+}$and $\mathbb{S}_{-}$are cells, interchanged by $\mathscr{A}$. The set $\mathbb{S}_{0}$ of spacelike directions forms an $\mathscr{A}$-invariant annulus bounded by the disjoint union $\partial \mathbb{S}_{+} \coprod \partial \mathbb{S}_{-}$.

Recall that $\mathbb{S}_{+}$is the Beltrami-Klein model of the hyperbolic plane where $\mathrm{SO}(2,1)^{o}$ acts as the orientation-preserving isometry group. Here the metric geodesics are precisely the projective geodesics and vice versa. (We will use the same term "geodesics" for both types of geodesics for ones in $\mathbb{S}_{+}$and later $\mathbb{S}_{-}$.) The geodesics in $\mathbb{S}_{+}$are straight arcs and $\partial \mathbb{S}_{+}$forms the ideal boundary of $\mathbb{S}_{+}$. For a finitely generated discrete subgroup $\Gamma$ in $\mathrm{SO}(2,1)^{\circ}$, the surface $\mathbb{S}_{+} / \Gamma$ has a complete hyperbolic structure as well as an $\mathbb{R P}^{2}$-structure with the compatible geodesic structure. 
Alternatively, we can use $\mathbb{S}_{-}$as the model and $\mathbb{S}_{-} / \Gamma$ has a complete hyperbolic structure as well as an $\mathbb{R P}^{2}$-structure. Since $\mathbb{S}_{+}$and $\mathbb{S}_{-}$are properly convex, these are examples of properly convex $\mathbb{R P}^{2}$-surfaces.

3.4. Null half-planes. Let $\mathcal{N}$ denote the nullcone in $\mathrm{V}$, that is, the collection of all vectors $\mathrm{v} \in \mathrm{V}$ with $\mathrm{v} \cdot \mathrm{v}=0$. Its projectivization $\mathrm{P}(\mathcal{N}-\{O\})$ consists of all null lines in $\mathrm{V}$. Suppose $\mathrm{v} \in \mathcal{N}-\{O\}$. Its orthogonal complement $\mathrm{v}^{\perp}$ is a null plane which contains the line $\mathbb{R v}$. The line $\mathbb{R} v$ separates $v^{\perp}$ into two half-planes.

The orientation on $\mathrm{V}$ determines an $\mathrm{SO}(2,1)$-invariant way to uniquely associate a component of $v^{\perp}-\mathbb{R} v$ to $\mathbb{R} v$ as follows. Since $v \in \mathcal{N}$ holds, its direction $((\mathrm{v}))$ lies in either $\partial \mathbb{S}_{+}$or $\partial \mathbb{S}_{-}$. Choose an arbitrary element $\mathrm{u}$ of $\mathbb{S}_{+}$or $\mathbb{S}_{-}$so that the directions of $\mathrm{v}$ and $\mathrm{u}$ both lie in $\mathrm{Cl}\left(\mathbb{S}_{+}\right)$or $\mathrm{Cl}\left(\mathbb{S}_{-}\right)$respectively. (For example $\mathrm{u}=(0,0, \pm 1)$ would be sufficient.) Define the null half-plane $\mathscr{W}(\mathrm{v})$ (or the wing) associated to $\mathrm{v}$ as:

$$
\mathscr{W}(\mathrm{v}):=\left\{\mathrm{w} \in \mathrm{v}^{\perp} \mid \operatorname{Det}(\mathrm{v}, \mathrm{w}, \mathrm{u})>0\right\} \subset \mathrm{v}^{\perp}-\mathbb{R} \mathrm{v} .
$$

Since the Lorentzian product $\mathrm{u} \times \mathrm{v}$ is in $\mathscr{W}(\mathrm{v})$, the directions in the wing satisfy the right-hand rule. Since $\mathscr{W}(v)=\mathscr{W}\left(v_{-}\right)$holds, the null halfplane $\mathscr{W}(\mathrm{v})$ depends only on $\mathbb{R} v$. The corresponding set of directions is the open arc

$$
\varepsilon(((\mathrm{v}))):=((\mathscr{W}(\mathrm{v})))
$$

in $\mathbb{S}_{0}$ joining $((\mathrm{v}))$ to its antipode $\left(\left(\mathrm{v}_{-}\right)\right)$. Since $\mathrm{v}^{\perp}$ is tangent to $\mathcal{N}$, the $\operatorname{arc} \varepsilon(((\mathrm{v})))$ is tangent to $\partial \mathbb{S}_{+}$. The orientation of $\partial \mathbb{S}_{+}$induced from $\mathbb{S}_{+}$agrees with the orientation of $\varepsilon(((\mathrm{v})))$ away from $((\mathrm{v}))$.

The corresponding map

$$
((\mathrm{v})) \longmapsto \varepsilon(((\mathrm{v})))
$$

is an $\mathrm{SO}(2,1)$-equivariant map

$$
\partial \mathbb{S}_{+} \rightarrow \mathcal{S}
$$

where $\mathcal{S}$ denotes the set of half-arcs of form $\varepsilon(((\mathrm{v})))$ for $((\mathrm{v})) \in \partial \mathbb{S}_{+}$. The $\operatorname{arcs} \varepsilon(((\mathrm{v})))$ for $((\mathrm{v})) \in \partial \mathbb{S}_{+}$foliate $\mathbb{S}_{0}$. We can obtain all arcs $\varepsilon(((\mathrm{v})))$ from $\varepsilon(((0: 1: 1)))$ by an $\mathbf{S}^{1}$-action fixing $(( \pm(0: 0: 1)))$ for a subgroup $\mathbf{S}^{1}$ of $\mathbf{S O}(2,1)$. Let us call the foliation $\mathcal{F}$.

Hence $\mathbb{S}_{0}$ has an $\mathbf{S O}(2,1)$-equivariant quotient map

$$
\mathbb{S}_{0} \stackrel{\Pi}{\rightarrow} \mathrm{P}(\mathcal{N}-\{O\}) \cong \mathbf{S}^{1}
$$

and $\varepsilon(((\mathrm{v})))=\Pi^{-1}([\mathrm{v}])$ for each $\mathrm{v} \in \mathcal{N}-\{O\}$.

Consider a future-pointing null vector of Euclidean length $\sqrt{2}$ :

$$
\mathrm{n}_{\theta}:=\left[\begin{array}{c}
\cos (\theta) \\
\sin (\theta) \\
1
\end{array}\right] \text { for } \theta \in[0,2 \pi)
$$


Then $\mathscr{W}\left(\mathrm{n}_{\theta}\right)$ consists of all

$$
\mathrm{p}_{\theta}(t, s):=t \mathrm{n}_{\theta}+s\left[\begin{array}{c}
-\sin (\theta) \\
\cos (\theta) \\
0
\end{array}\right] \text { for } t \in \mathbb{R}, s>0
$$

The $\operatorname{arc} c_{\theta}:=\varepsilon\left(\left(\left(\mathrm{n}_{\theta}\right)\right)\right)$ on the sphere of directions $\mathbf{S}_{\infty}^{2}$ is parametrized by unit vectors

$$
\frac{1}{\sqrt{2}} \mathrm{p}_{\theta}\left(t, \sqrt{1-t^{2}}\right) \text { as }-1<t<1 .
$$

(As an element of $\mathbf{S}^{3}$, its homogeneous coordinates equal

$$
\left(\left(\frac{1}{\sqrt{2}} \mathrm{p}_{\theta}\left(t, \sqrt{1-t^{2}}\right): 0\right)\right)
$$

where $\frac{1}{\sqrt{2}} \mathrm{p}_{\theta}\left(t, \sqrt{1-t^{2}}\right)$ is used as the first three coordinates.)

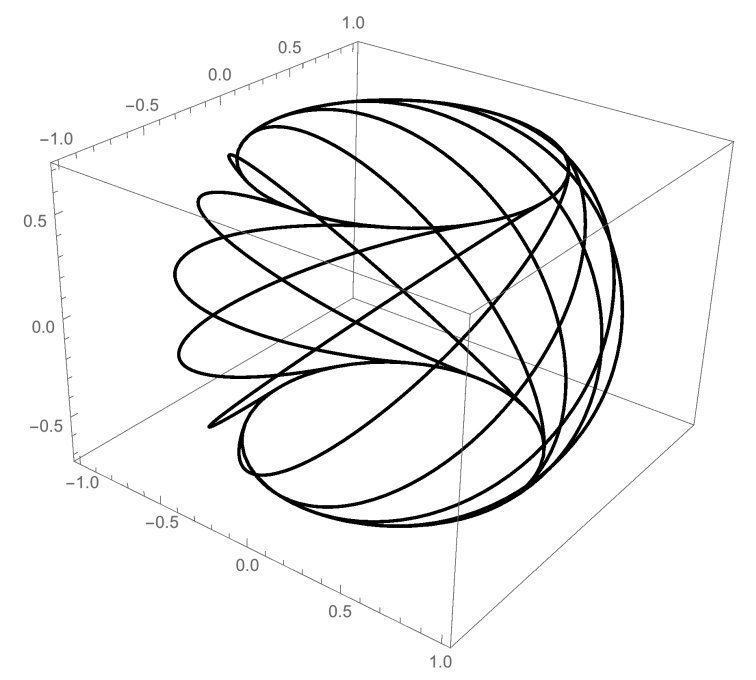

Figure 1. The tangent geodesics to disks $\mathbb{S}_{+}$and $\mathbb{S}_{-}$in the unit sphere $\mathbf{S}_{\infty}^{2} \quad$ embedded in $\mathbb{R}^{3}$ as mapped by the normalization map of (1).

The other component of $\mathbf{v}^{\perp}-\mathbb{R} v$ could also be used as a wing $\mathscr{W}(\mathrm{v})$, but for the opposite orientation on $\mathrm{V}$. Alternatively, these negatively oriented null half-planes are the images of the positively oriented null half-planes under the antipodal map $\mathscr{A}$ (which reverses orientation in dimension three). This phenomenon appears in the theory of crooked planes, where one must choose a class of orientations for crooked planes, as well as the Margulis invariant, where the choice of orientation plays a crucial role.

Since we have no need to consider negatively oriented null halfplanes, we henceforth restrict our attention to positively oriented null half-planes in this paper. 


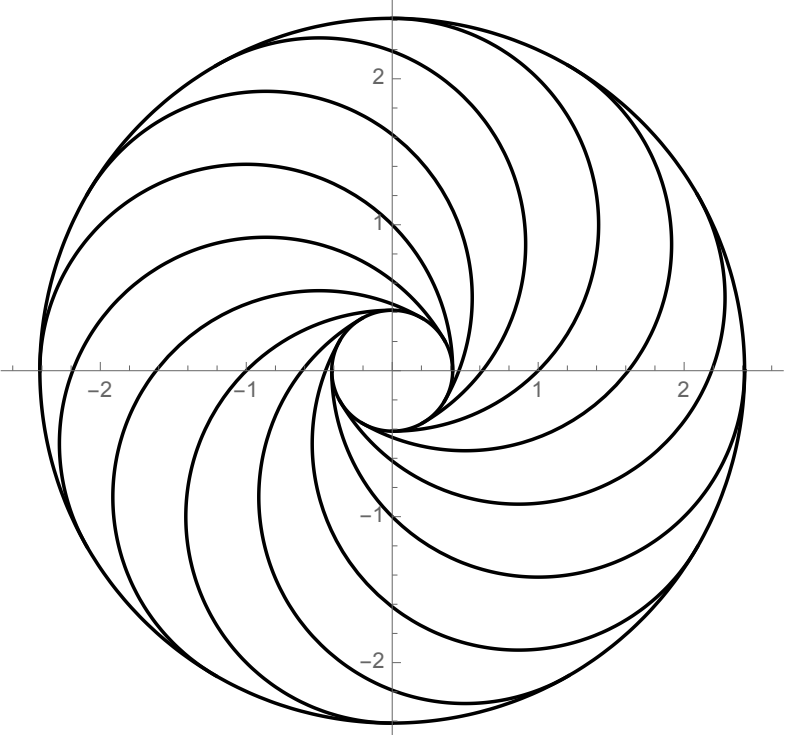

Figure 2 .

The tangent geodesics to disks $\mathbb{S}_{+}$and $\mathbb{S}_{-}$in the stereographically projected $\quad \mathbf{S}_{\infty}^{2}$ from $(0,0,-1)$. Radial arcs are geodesics. The inner circle represents the boundary of $\mathbb{S}_{+}$. The arcs of form $\varepsilon(x)$ for $x \in \partial \mathbb{S}_{+}$ are leaves of the foliation $\mathcal{F}$ on $\mathbb{S}_{0}$.

\subsection{Affine boosts.}

Definition 3.1. An isometry of $\mathrm{E}$ is an (affine) boost if its linear part is a positive hyperbolic element of $\mathrm{SO}(2,1)$ and it acts freely on $\mathrm{E}$.

Suppose that $\gamma \in \operatorname{Isom}^{+}(\mathrm{E})$ is an affine boost. Then $\gamma$ preserves a unique line in $E$ which we denote $\operatorname{Axis}(\gamma)$. Furthermore $\operatorname{Axis}(\gamma)$ is spacelike and the restriction of $\gamma$ to $\operatorname{Axis}(\gamma)$ is a nonzero translation. In a suitable coordinate system, we can take $\operatorname{Axis}(\gamma)$ to be the $y$-axis, in which case $\gamma$ is the affine transformation:

$$
p \stackrel{\gamma}{\longmapsto}\left[\begin{array}{ccc}
e^{l} & 0 & 0 \\
0 & 1 & 0 \\
0 & 0 & e^{-l}
\end{array}\right] p+\left[\begin{array}{l}
0 \\
\alpha \\
0
\end{array}\right]
$$

where

$$
\left[\begin{array}{ccc}
e^{l} & 0 & 0 \\
0 & 1 & 0 \\
0 & 0 & e^{-l}
\end{array}\right]
$$

is the linear part $\mathbb{L}(\gamma)$ of $\gamma$ and $p \in \mathrm{E}$. We say that $\gamma$ is in standard form if $\operatorname{Axis}(\gamma)$ is the $y$-axis. In that case $\gamma$ is given by (3) as above.

3.5.1. Oriented axes. As observed by Margulis [53], the axis of an affine boost $\gamma$ admits a canonical orientation, induced by the ambient orientation of $\mathrm{E}$.

Let $g=\mathbb{L}(\gamma) \in \mathbf{S O}(2,1)$ be positive hyperbolic. Then since $g$ is an isometry, and unimodular, the eigenvalues of $g$ are $\lambda, 1, \lambda^{-1}$ where $\lambda>1$. The eigenspaces for eigenvalues $\lambda, \lambda^{-1}$ are null (since $g$ is an 
isometry); choose respective eigenvectors $\mathbf{v}_{+}(g), \mathbf{v}_{-}(g) \in \mathrm{V}$ with the same causal character (that is, they are either both future-pointing or both past-pointing). The 1-eigenspace $\mathrm{v}_{0}(g) \subset \mathrm{V}$ is spacelike and orthogonal to $\mathbf{v}_{+}(g)$ and $\mathbf{v}_{-}(g)$. We define $\mathbf{v}_{+}(\gamma):=\mathbf{v}_{+}(g), \mathbf{v}_{0}(\gamma):=$ $\mathrm{v}_{0}(g), \mathbf{v}_{-}(\gamma):=\mathbf{v}_{-}(g)$.

Definition 3.2. The neutral eigenvector of $\gamma$ is the unique unit-spacelike eigenvector $\mathbf{v}_{0}(\mathbb{L}(\gamma))$ such that $\left\{\mathbf{v}_{+}(\gamma), \mathbf{v}_{0}(\gamma), \mathbf{v}_{-}(\gamma)\right\}$ is a positively oriented basis of $\mathrm{V}$. (See [34] for details.)

Since $\operatorname{Axis}(\gamma)$ is parallel to $\mathbf{v}_{0}(\mathbb{L}(\gamma))$, the neutral eigenvector $\mathbf{v}_{0}(\gamma)$ of $\mathbb{L}(\gamma)$ defines an orientation on $\operatorname{Axis}(\gamma)$. Denote the corresponding oriented geodesic by $\widehat{\operatorname{Axis}}(\gamma)$. By $(3)$, the restriction of $\gamma$ to $\operatorname{Axis}(\gamma)$ is a translation. Since $\operatorname{Axis}(\gamma)$ is spacelike, the displacement of $\gamma$ along $\widehat{\operatorname{Axis}}(\gamma)$ is a well-defined real number

$$
\mu(\gamma):=(\gamma(p)-p) \cdot \mathrm{v}_{0}(\gamma)=\alpha \in \mathbb{R}, p \in \mathbf{E},
$$

called the Margulis invariant. For the basic properties of this invariant, see [53], 46], [1], [14], [15].

3.5.2. Invariant planes. The weak-stable plane $W^{w s}(\gamma) \subset \mathrm{E}$ is the affine subspace containing $\operatorname{Axis}(\gamma)$ and parallel to the plane spanned by $\mathrm{v}_{0}(\gamma)$ and $\mathrm{v}_{-}(\gamma)$. Points in this affine plane asymptotically approach $\operatorname{Axis}(\gamma)$ : If $p \in W^{w s}(\gamma)$, then

$$
\begin{aligned}
\mathbf{d}\left(\gamma^{n}(p),\left(\left(\mathrm{v}_{0}(\gamma): 0\right)\right)\right) & \rightarrow 0, \\
\mathbf{d}\left(\gamma^{n}(p), \text { Axis }(\gamma)\right) & \rightarrow 0
\end{aligned}
$$

as $n \rightarrow \infty$.

3.5.3. Classification of affine boosts. An affine boost $\gamma$ is completely determined by the oriented spacelike line $\widehat{A x i s}(\gamma)$ and the two real numbers $\ell>0$ and $\alpha \neq 0$. Its conjugacy class is determined by the pair $(\ell, \alpha) \in \mathbb{R}^{2}$.

\subsection{Projective boosts.}

Definition 3.3. A projective boost is a collineation of $\mathbf{S}^{3}$ which extends an affine boost $g$ on $\mathrm{E}$. Namely, an affine boost $\mathrm{E} \stackrel{g}{\rightarrow} \mathrm{E}$ extends to a projective transformation of $\mathbb{P}^{3}$ and lifts to a collineation $\gamma$ of the double covering $\mathbf{S}^{3}$. 
3.6.1. Standard form of a projective boost. A standard projective boost is the extension of an affine boost in standard form to $\mathbf{S}^{3}$. Explicitly, $\gamma$ is given by a $4 \times 4$-matrix in a suitable coordinate system

$$
\left[\begin{array}{cccc}
e^{\ell} & 0 & 0 & 0 \\
0 & 1 & 0 & \alpha \\
0 & 0 & e^{-\ell} & 0 \\
0 & 0 & 0 & 1
\end{array}\right]
$$

where $\ell>0$ and $\alpha \neq 0$, which will be related to the Margulis invariant. It restricts to the affine boost defined by (3) on the affine patch

$$
\left[\begin{array}{l}
x \\
y \\
z
\end{array}\right] \longmapsto((x: y: z: 1)) \text {. }
$$

The six fixed points on $\mathbf{S}_{\infty}^{2}$ are:

$$
\begin{aligned}
& x_{ \pm}^{+}:=(( \pm 1: 0: 0: 0)), \\
& x_{ \pm}^{0}:=((0: \pm 1: 0: 0)), \\
& x_{ \pm}^{-}:=((0: 0: \pm 1: 0)) .
\end{aligned}
$$

in homogeneous coordinates on $\mathbf{S}_{\infty}^{2}$. For $l \neq 0, \alpha \neq 0$, these are the only fixed points on $\mathbf{S}^{3}$. Also, $\gamma$ acts on the subspace $\sigma:=[0: *: 0: *]$.

Such a collineation induces the affine isometry of $\mathrm{E}$ defined by (3). For a given projective boost, denote by $x^{+}(\gamma), x^{0}(\gamma), x^{-}(\gamma)$, and $\sigma(\gamma)$ the points going to $x^{+}, x^{0}, x^{-}$and the subspace $\sigma$ when $\gamma$ is put in standard form. The span

$$
\mathscr{W}^{w s}(\gamma):=\operatorname{span}\left(x^{-}(\gamma) \cup \sigma(\gamma)\right)
$$

is the subspace of $\mathbf{S}^{3}$ extending the weak-stable plane $W^{w s}(\gamma) \subset \mathbf{E}$. Under the action of $\gamma^{n}$ as $n \rightarrow+\infty$, images of points on $W^{w s}(\gamma) \subset \mathbf{E}$ limit towards $x^{0}(\gamma)$ along the line

$$
\sigma(\gamma)-\left\{x^{0}(\gamma), x^{0}(\gamma)_{-}\right\}
$$

where $\sigma(\gamma)$ is the subspace spanned by $\operatorname{Axis}(\gamma)$ in $\mathbf{E}$.

3.6.2. The action of projective-boost automorphisms. Recall that $\mathbf{S}^{3}$ is the quotient $\mathbb{R}^{4}-\{O\} / \sim$ where $\sim$ is given by $\mathrm{v} \sim \mathrm{w}$ iff $\mathrm{v}=s \mathrm{w}$ for $s>0$. Recall that homogeneous coordinate system of $\mathbf{S}^{3}$ is given by setting a point $p$ of $\mathbf{S}^{3}$ to have coordinates $((x: y: z: t))$ where $(x, y, z, t)$ divided by its Euclidean norm represent $p$ as a unit vector in $\mathbf{S}^{3}$. We identified $\mathrm{E}$ with the open affine space given by $t>0$. We use the coordinates so that $\mathbf{S}_{\infty}^{2}$ corresponds to the hyperplane given by $t=0$ and the origin $O$ of $\mathrm{E}$ is given by $\mathrm{e}_{4}$. The closure of $\mathrm{E}$ is the 3-dimensional hemisphere $\mathscr{H}$ with $\partial \mathscr{H}=\mathbf{S}_{\infty}^{2}$. For this system of coordinates, $(x, y, z) \in \mathrm{E}$ is 


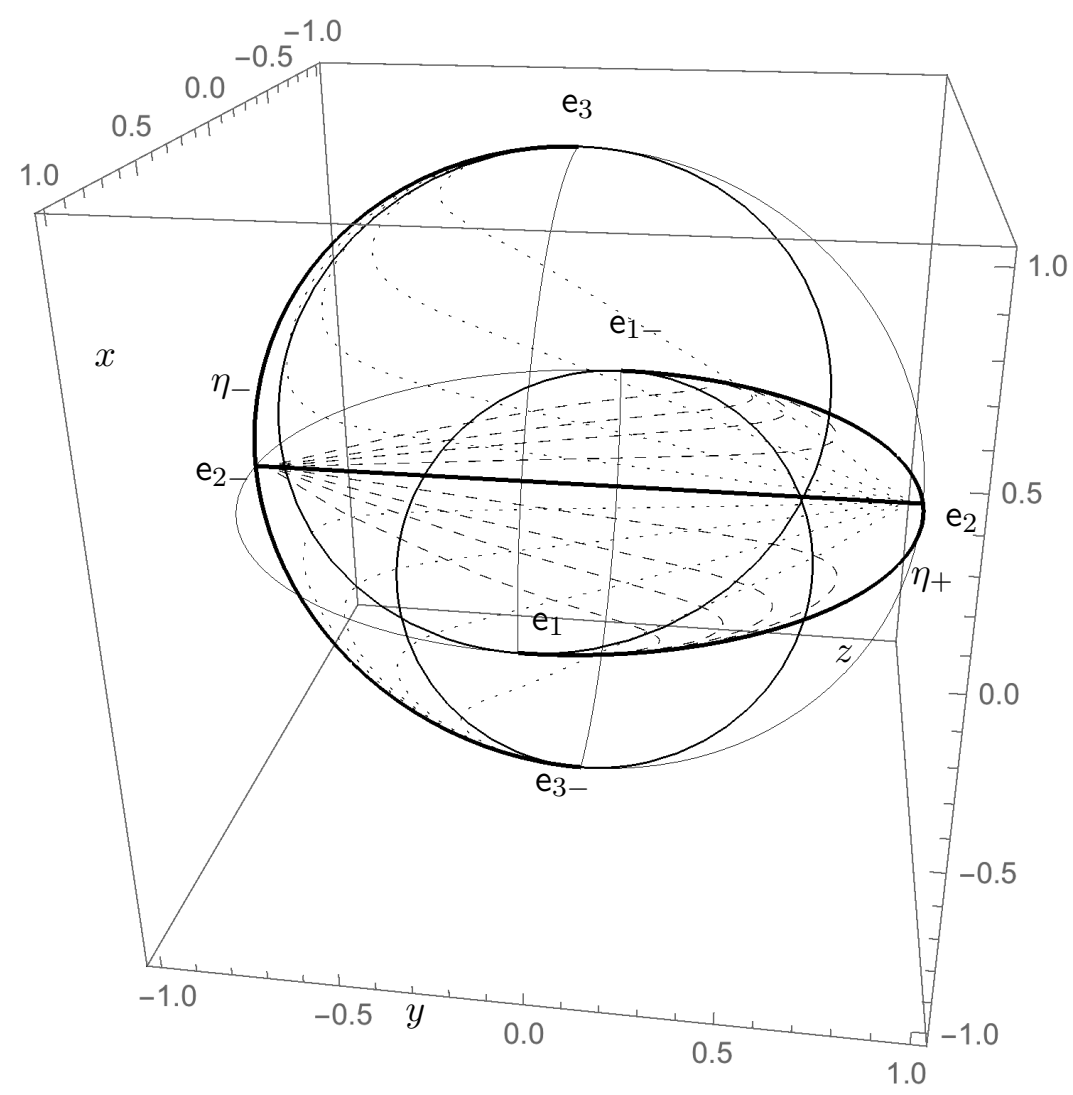

Figure 3. The action of a projective boost $\hat{g}$ on the 3-hemisphere $\mathscr{H}$ where the boundary sphere $\mathbf{S}_{\infty}^{2}$ is the unit sphere with center $(0,0,0)$ here as mapped by the normalization map (1). The mathematica file is given at [19].

given homogeneous coordinates $((x: y: z: 1))$ and coordinates in $\mathbf{S}_{\infty}^{2}$ denoted $((x: y: z: 0))$.

Recall from $\$ 3.6$, a projective automorphism $g$ of form

$$
\left[\begin{array}{cccc}
\lambda & 0 & 0 & 0 \\
0 & 1 & 0 & k \\
0 & 0 & \frac{1}{\lambda} & 0 \\
0 & 0 & 0 & 1
\end{array}\right] \lambda>1, k \neq 0
$$

under a homogeneous coordinate system of $\mathbf{S}^{3}$ is a projective-boost. We call the above matrix the standard boost matrix with parameters $(\lambda, k)$. In this coordinate system, $g$ acts on an open 3-dimensional hemisphere, to be identified with $\mathrm{E}$ given by $t>0$, leaving invariant the following 
subspaces:

$$
\begin{aligned}
\operatorname{Axis}(g) & =\sigma_{g} \cap \mathrm{E}, & \sigma_{g}:=\{((x: y: z: t)) \mid x=0, z=0\}, \\
W^{w s}(g) & =S_{g} \cap \mathrm{E}, & \mathscr{W}^{w s}=S_{g}:=\{((x: y: z: t)) \mid x=0\}, \\
W^{w u}(g) & =U_{g} \cap \mathrm{E}, & U_{g}:=\{((x: y: z: t)) \mid z=0\} .
\end{aligned}
$$

On $S_{g} \cap \mathscr{H}^{\circ}, g$ contracts vectors along a direction by a constant $1 / \lambda$ and $g$ expands the vectors along a direction by a constant $\lambda$ on

$$
\mathscr{W}^{w s}(g) \cap \mathscr{H}^{o}=W^{w s}(g)
$$

assuming $\lambda>1$. The subspace $S_{g}$ given by $x=0$ is the weak-stable subspace, and the subspace $U_{g}$ given by $z=0$ is the weak-unstable subspace. (See \$3.6.1.)

The great semicircles

$$
\begin{aligned}
& \eta_{+}:=\overline{\mathrm{e}_{1} \mathrm{e}_{2}\left(\mathrm{e}_{1-}\right)} \\
& \eta_{-}:=\overline{\mathrm{e}_{3}\left(\mathrm{e}_{2-}\right)\left(\mathrm{e}_{3-}\right)}
\end{aligned}
$$

are the the attracting arc, and repelling arc for $g$, respectively. Their endpoints $\mathrm{e}_{1}, \mathrm{e}_{1-}, \mathrm{e}_{3}, \mathrm{e}_{3-}$ lie on the null latitudes of $\mathbf{S}_{\infty}^{2}$, and both $\eta_{+}^{o}$ and $\eta_{-}^{o}$ are fibers of the collapsing map

$$
\mathbb{S}_{0} \stackrel{\varepsilon}{\rightarrow} \mathbb{R} P^{1}
$$

3.7. Sequences of projective boosts. Let $\left\{\gamma_{n}\right\}$ be a sequence of projective boosts in Aut $\left(\mathbf{S}^{3}\right)$. A rank of an element of $\mathbb{S}\left(\operatorname{Mat}_{4}(\mathbb{R})\right)$ is the rank of any linear map $\mathbb{R}^{4} \rightarrow \mathbb{R}^{4}$ representing it. The compactness of $\mathbb{S}\left(\operatorname{Mat}_{4}(\mathbb{R})\right)$ implies that convergent subsequences exist. Suppose that

$$
\gamma_{\infty}=\lim _{n \rightarrow+\infty} \gamma^{n}
$$

is a projective endomorphism in $\mathbb{S}\left(\operatorname{Mat}_{4}(\mathbb{R})\right.$ ) of rank one. (In general, the limit might have rank 2 or 3.) Then the undefined hyperplane of $\gamma_{\infty}$ equals a subspace $\mathscr{W}_{\infty}^{w s}$ of codimension one and the image of $\gamma_{\infty}$ equals the pair $x_{\infty}^{+}, x_{\infty-}^{+}$where

$$
\begin{gathered}
\mathscr{W}^{w s}\left(\gamma_{n}\right) \longrightarrow \mathscr{W}_{\infty}^{w s}, \\
x^{+}\left(\gamma_{n}\right) \longrightarrow x_{\infty}^{+}
\end{gathered}
$$

as $n \longrightarrow+\infty$.

More generally, suppose that $\left\{\gamma_{n}\right\}$ is a sequence in Aut $\left(\mathbf{S}^{3}\right)$ converging to a projective endomorphism $\gamma_{\infty}$ in $\mathbb{S}\left(\operatorname{Mat}_{4}(\mathbb{R})\right)$ of rank one. Let $N\left(\gamma_{\infty}\right)$ denote the kernel of $\gamma_{\infty}$. We can choose a subsequence of $\left\{\gamma_{n}\right\}$ so that the following hold:

- the corresponding subsequence of the attracting fixed points $\left\{x^{+}\left(\gamma_{n}\right)\right\}$ converges to a point in the image $I\left(\gamma_{\infty}\right)$ and, 
- the corresponding subsequence of the extended weak-stable subspaces $\left\{\mathscr{W}^{w s}\left(\gamma_{n}\right)\right\}$ converges to the undefined subspace $\mathbb{S}\left(N\left(\gamma_{\infty}\right)\right)$.

3.7.1. Convergence Lemma. We will prove the following lemma for a properly convex $K$ because the proof is much simpler. But this is true for a general compact subset $K$ by the proof of Proposition 6.2.

Lemma 3.4. Let $g_{\lambda, k}$ denote the projective boost on $\mathscr{H}$ defined by the standard boost matrix in (5) for homogeneous coordinates with coordinate functions $x, y, z, t$ and let $\mathbf{S}_{0}^{2}=\mathscr{W}^{w s}\left(g_{\lambda, k}\right)$ denote the subspace given by $x=0$. We assume that $k \geq 0, \lambda>0$. Then as $\lambda, k \rightarrow+\infty$ where $k / \lambda \rightarrow 0$ :

(a) $g_{\lambda, k} \mid \mathscr{H}-\mathbf{S}_{0}^{2}$ converges in the compact-open topology to a projective endomorphism

$$
((x: y: z: t)) \stackrel{\Pi_{0}}{\longrightarrow}((\operatorname{sgn}(x): 0: 0: 0))=\mathrm{e}_{1} \text { or } \mathrm{e}_{1-}, t \geq 0 .
$$

(b) $g_{\lambda, k} \mid\left(\mathbf{S}_{0}^{2} \cap \mathscr{H}\right)-\eta_{-}$converges to a projective endomorphism

$$
((0: y: z: t)) \stackrel{\Pi_{1}}{\longrightarrow} \mathrm{e}_{2} .
$$

(c) For a properly convex compact set $K$ in $\mathscr{H}-\eta_{-}$, a subsequence of $\left\{g_{\lambda, k}(K)\right\}$ converges to

$-\eta_{+}=\overline{\mathrm{e}_{1} \mathrm{e}_{2} \mathrm{e}_{1-}}$ provided $K$ meets both components of

$$
\mathscr{H}-\left(\mathbf{S}_{0}^{2} \cap \mathscr{H}\right)
$$

- otherwise to

(i) $\left\{\mathrm{e}_{2}\right\}$ if $K \subset\left(\mathbf{S}_{0}^{2} \cap \mathscr{H}\right)-\eta_{-}$,

(ii) $\left\{\mathrm{e}_{1}\right\}$ or $\left\{\mathrm{e}_{1-}\right\}$ if $K \cap\left(\mathbf{S}_{0}^{2} \cap \mathscr{H}\right)=\emptyset$, or else

(iii) one of the segments

$$
\overline{\mathrm{e}_{1} \mathrm{e}_{2}}, \overline{\mathrm{e}_{1-} \mathrm{e}_{2}} \text {. }
$$

Proof. The first two items follow by normalizing the above matrix by dividing it by the maximal norm of the entries.

So, we now suppose that $K \cap \mathbf{S}_{0}^{2} \neq \emptyset$, and $K$ meets both components of $\mathscr{H}-\mathbf{S}_{0}^{2}$. For a properly convex compact subset $K$ in $\mathscr{H}-\eta_{-}$, let $K_{1}$ be the nonempty properly convex compact set $K \cap \mathbf{S}_{0}^{2}-\eta_{-}$. We can take a closed 2-dimensional hemisphere $H$ with boundary $\partial H \subset \mathbf{S}_{\infty}^{2}$ so that a component $H^{\prime}$ of $\mathscr{H}-H$ satisfies

$$
\begin{aligned}
K & \subset H^{\prime}, \\
H^{\prime} \cap \eta_{-} & =\emptyset, \\
H & =\partial_{\mathscr{H}} H^{\prime}
\end{aligned}
$$

which is the boundary of $H^{\prime}$ in the relative topology of $\mathscr{H}$.

We can operate as below: 
- there exist a properly convex compact domain $D_{1}$ in a component of $H^{\prime}-\mathbf{S}_{0}^{2}$ with $x>0$ and another one $D_{2}$ in a component of $H^{\prime}-\mathbf{S}_{0}^{2}$ with $x<0$ so that

$$
\begin{aligned}
& K \subset J:=\operatorname{Join}\left(D_{1}, D_{2}\right), \\
& J \cap \eta_{-}=\emptyset .
\end{aligned}
$$

- For

$$
K_{2}:=J \cap \mathbf{S}_{0}^{2} \supset K_{1},
$$

the join $J_{1}:=\operatorname{Join}\left(K_{2}, D_{1}\right)$ and the join $J_{2}:=\operatorname{Join}\left(K_{2}, D_{2}\right)$ satisfy $J=J_{1} \cup J_{2}$.

By the second item of Lemma 3.4, $\left\{g_{\lambda, k}\left(K_{2}\right)\right\}$ converges to $\mathrm{e}_{2}$ as $\lambda, k \rightarrow \infty$. Since

$$
\left\{g_{\lambda, k}\left(D_{1}\right)\right\} \rightarrow\left\{\mathrm{e}_{1}\right\} \text { and }\left\{g_{\lambda, k}\left(D_{2}\right)\right\} \rightarrow\left\{\mathrm{e}_{1-}\right\}
$$

as $\lambda, k \rightarrow \infty$ by above,

- $\left\{g_{\lambda, k}\left(J_{1}\right)\right\} \rightarrow s_{1}=\overline{\mathrm{e}_{1} \mathrm{e}_{2}}$ in $\mathbf{S}_{\infty}^{2}$ and

- $\left\{g_{\lambda, k}\left(J_{2}\right)\right\} \rightarrow s_{2}=\overline{\mathrm{e}_{1-} \mathrm{e}_{2}}$ in $\mathbf{S}_{\infty}^{2}$ hold by Lemma 2.2 respectively.

Hence, we conclude $\left\{g_{\lambda, k}(J)\right\} \rightarrow \eta_{+}$.

Let $p_{1} \in K_{1}$ and let $p_{+} \in D_{1}, p_{-} \in D_{2}$ be two points. Then

$$
\left\{g_{\lambda, k}\left(\overline{p_{+} p_{1}} \cup \overline{p_{-} p_{1}}\right)\right\} \rightarrow \eta_{+} \text {as } \lambda, k \rightarrow \infty \text {. }
$$

Since

$$
\overline{p_{+} p_{1}} \cup \overline{p_{-} p_{1}} \subset K \subset J
$$

we obtain $\left\{g_{\lambda, k}(K)\right\} \rightarrow \eta_{+}$as $\lambda, k \rightarrow \infty$ by Section 2.4 .

So, we now suppose that $K \cap \mathbf{S}_{0}^{2} \neq \emptyset$ and $K$ meets only one component of $\mathscr{H}-\mathbf{S}_{0}^{2}$. Then the arguments are simpler and $\left\{g_{\lambda, k}(K)\right\}$ converges to $\overline{\mathrm{e}_{1} \mathrm{e}_{2}}$ or $\overline{\mathrm{e}_{1-} \mathrm{e}_{2}}$ as $\lambda, k \rightarrow \infty$.

We remark that our proof of Proposition 6.2 generalizes the above one, using dynamical properties of Margulis spacetimes.

\section{Dynamics AND hyperbolic GeOMETRY}

4.1. $\mathbb{R P}^{2}$-structures on surfaces. Let $\vartheta$ correspond to an element $A$ of $\mathrm{SL}(3, \mathbb{R})$ diagonalizable with three distinct positive eigenvalues. Then the induced $\vartheta^{\prime} \in \operatorname{Aut}\left(\mathbf{S}_{\infty}^{2}\right)$ has six fixed points $a, a_{-}, r, r_{-}, s, s_{-}$ and three great circles $l_{1}, l_{2}$, and $l_{3}$ so that

$$
\begin{aligned}
& \left\{a, r, a_{-}, r_{-}\right\} \subset l_{1}, \\
& \left\{r, s, r_{-}, s_{-}\right\} \subset l_{2}, \\
& \left\{a, s, a_{-}, s_{-}\right\} \subset l_{3} .
\end{aligned}
$$


We assume that $a$ corresponds to the largest eigenvalue and $r$ to the smallest one. Eight invariant open triangles compose $\mathbf{S}_{\infty}^{2}-\left(l_{1} \cup l_{2} \cup l_{3}\right)$. Let $\triangle$ be the triangle with vertices $a, r, s$. Then

$$
\begin{aligned}
& \left(\triangle \cup \overline{a r}^{o} \cup \overline{a s}^{o}\right) /\left\langle\vartheta^{\prime}\right\rangle, \\
& \left(\triangle \cup \overline{a r}^{o} \cup \overline{r s} \bar{s}^{o}\right) /\left\langle\vartheta^{\prime}\right\rangle
\end{aligned}
$$

are both examples of compact annuli. As quotients of domains of $\mathbf{S}_{\infty}^{2}$, they have $\mathbb{R} P^{2}$-structures. (See $\$ 2.1$.) Such an $\mathbb{R} P^{2}$-surface-withboundary is called an elementary annulus. Let $\triangle^{\prime}$ be an adjacent triangle sharing $\overline{a r}^{o}$ with $\triangle$ in the boundary, and $\triangle^{\prime \prime}$ be one sharing $\overline{a s}^{o}$ with $\triangle$ in the boundary. Then

$$
\begin{aligned}
& \left(\triangle \cup \triangle^{\prime} \cup \overline{a r}^{o} \cup \overline{a s}^{o} \cup{\overline{a s_{-}}}^{o}\right) /\left\langle\vartheta^{\prime}\right\rangle, \\
& \left(\triangle \cup \triangle^{\prime \prime} \cup \overline{a r}^{o} \cup{\overline{a r_{-}}}^{o} \cup \overline{a s}^{o}\right) /\left\langle\vartheta^{\prime}\right\rangle
\end{aligned}
$$

are examples of compact annuli again. An $\mathbb{R P}^{2}$-surface projectively diffeomorphic to one of these (for a choice of $\vartheta$ as above) is said to be a $\pi$-annulus. They are the union of two elementary annuli meeting at a boundary component. (See [20] and [21] for more details.)

Let $\tilde{S}$ be the universal cover of an $\mathbb{R P}^{2}$-surface $S$ with a developing map dev : $\tilde{S} \rightarrow \mathbb{R} P^{2}$ and a holonomy homomorphism $h$. For a circle $\mathbf{S}^{1}$, a closed geodesic $c: \mathbf{S}^{1} \rightarrow S$ is a closed curve where dev o $\tilde{c}$ is a straight arc in $\mathbb{R P}^{2}$ for a lift $\tilde{c}: \mathbb{R} \rightarrow \tilde{S}$ of $c$. A closed geodesic is principal if for a lift $\tilde{c}$ to the universal cover $\tilde{S}$, $\operatorname{dev} \circ \tilde{c}$ is an embedding to a straight arc connecting an attracting and a repelling fixed point of $h(\gamma)$ for a deck transformation $\gamma$ of $\tilde{S}$ satisfying $\tilde{c}(t+2 \pi)=\gamma \circ \tilde{c}(t)$. (Here $\gamma$ is said to be the corresponding deck transformation of $\tilde{c}$ and $c$, and it exists uniquely.)

A disjoint collection $c_{1}, \ldots, c_{m}$ of simple closed geodesics decomposes an $\mathbb{R P}^{2}$-surface $S$ into subsurfaces $S_{1}, . ., S_{n}$ if each $S_{i}$ is the closure of a component of $S-\bigcup_{i=1, ., m} c_{i}$ where we do not allow a curve $c_{i}$ to have two one-sided neighborhoods in only one $S_{j}$ for some $j$.

In [21], we proved:

Theorem 4.1. Let $\Sigma$ be a compact orientable $\mathbb{R P}^{2}$-surface with principal geodesic or empty boundary and $\chi(\Sigma)<0$. Then $\Sigma$ has a collection of disjoint simple closed principal geodesics decomposing $\Sigma$ into properly convex $\mathbb{R P}^{2}$-surfaces with principal geodesic boundary and of negative Euler characteristic and/or $\pi$-annuli with principal geodesic boundary.

4.2. Complete hyperbolic surfaces and Fuchsian groups. Let $G$ be a discrete subgroup of projective automorphisms of $\mathbb{S}_{+} \operatorname{in} \operatorname{Aut}\left(\mathbf{S}_{\infty}^{2}\right)$. We assume that $G$ has no elliptic or parabolic elements. Here complete 
geodesics of the hyperbolic metrics are maximal straight lines in $\mathbb{S}_{+}$and vice versa.

We suppose that $G$ is nonelementary; that is, it does not act on a pair of points in $\mathrm{Cl}\left(\mathbb{S}_{+}\right)$. Then $\mathbb{S}_{+} / G$ has a complete hyperbolic metric induced from the Hilbert one. $\mathbb{S}_{+} / G$ is geometrically finite in the sense that $\mathbb{S}_{+} / G$ contains a compact surface bounded by closed geodesics that is the deformation retract of $\mathbb{S}_{+} / G$.

Let $\Lambda$ denote the limit set of an orbit $G(x)$ for a point $x \in \mathbb{S}_{+}$, Since $G$ is a geometrically finite Fuchsian group, $\Lambda$ is a Cantor subset of $\partial \mathbb{S}_{+}$ of Lebesgue measure zero and contains the dense set of fixed points of nonidentity elements of $G$. Let us define $\Sigma_{+}:=\mathbb{S}_{+} / G$. We define $\tilde{\Sigma}_{+}^{\prime}$ as $\mathrm{Cl}\left(\mathbb{S}_{+}\right)-\Lambda$. (See Chapter 8 of Beardon [5] for the classical Fuchsian group theory used here.)

Let $\mathcal{J}$ be the set indexing the boundary components of $\tilde{\Sigma}^{\prime}$. We denote the boundary components of $\tilde{\Sigma}_{+}^{\prime}$ by $\partial_{i} \mathbb{S}_{+}, i \in \mathcal{J}$. Hence,

$$
\tilde{\Sigma}_{+}^{\prime}=\mathbb{S}_{+} \cup \bigcup_{i \in \mathcal{J}} \partial_{i} \mathbb{S}_{+}=\mathrm{Cl}\left(\mathbb{S}_{+}\right)-\Lambda
$$

We obtain a compact surface $\Sigma_{+}^{\prime}=\tilde{\Sigma}_{+}^{\prime} / G$ containing open surface $\Sigma_{+}$ compactifying $\Sigma_{+}$with boundary components diffeomorphic to circles.

Note that for each element $g \in G$, either $g\left(\partial_{i} \mathbb{S}_{+}\right) \cap \partial_{i} \mathbb{S}_{+}=\emptyset$ or $g\left(\partial_{i} \mathbb{S}_{+}\right)=\partial_{i} \mathbb{S}_{+}$holds with $g$ in the cyclic group generated by the deck transformation corresponding to $\partial_{i} \mathbb{S}_{+}$.

We obtain a convex domain $\Omega_{+}$closed in $\mathbb{S}_{+}$bounded by a union of straight segments $\mathbf{l}_{i}$ for $i \in \mathcal{J}$. Each $\mathbf{l}_{i}$ is a geodesic in $\mathbb{S}_{+}$connecting ideal endpoints of $\partial_{i} \mathbb{S}_{+}$. We choose a primitive element $\mathbf{g}_{i} \in \Gamma$ acting on $\mathbf{l}_{i}$ for each $i \in \mathcal{J}$. Since $\Gamma$ acts as a geometrically finite Fuchsian group on $\mathbb{S}_{+}$without parabolics, $\Omega_{+} / \Gamma$ is a compact hyperbolic surface with geodesic boundary that is the convex hull of $\mathbb{S}_{+} / \Gamma$ and homeomorphic to $\Sigma_{+}^{\prime}$. Thus,

$$
\mathbb{S}_{+}-\Omega_{+}=\coprod_{i \in \mathcal{J}} D_{i}
$$

where $D_{i}$ is a convex open domain $\partial D_{i}=\mathrm{Cl}\left(\partial_{i} \mathbb{S}_{+}\right) \cup \mathbf{l}_{i}$ for $i \in \mathcal{J}$ that covers an open annulus in $\Sigma_{+}^{\prime}$.

Since $\mathbf{l}_{i}$ covers a simple closed geodesic in $\Sigma_{+}$,

$$
\begin{aligned}
& g\left(\mathbf{l}_{i}\right) \cap \mathbf{l}_{i}=\emptyset \text { if } i \neq j, g \in \Gamma, \\
& g(\mathbf{l})_{i} \cap \mathbf{l}_{i}=\emptyset \text { if } g \notin \operatorname{Stab}\left(l_{i}\right) .
\end{aligned}
$$

4.3. The dynamics of geodesic flows on hyperbolic surfaces. Now we assume that $\Gamma$ also acts freely and properly on E. Assume that $\mathbb{L}(\Gamma) \subset \mathrm{SO}(2, \mathbb{R})^{o}$. We recall that $\Gamma$ is isomorphic to a free group of finite rank $\geq 2$, and $\mathbb{S}_{+} / \Gamma$ is a complete genus $\tilde{\mathbf{g}}$ hyperbolic surface 
with b ideal boundary components and without parabolics. Recall that $\Sigma_{+}$is the interior of the surface $\Sigma_{+}^{\prime}$.

The Fuchsian $\Gamma$-action on the boundary $\partial \mathbb{S}_{+}$of the standard disk $\mathbb{S}_{+}$in $\mathbf{S}_{\infty}^{2}$ forms a discrete convergence group: For every sequence $g_{j}$ of mutually distinct elements of $\Gamma$, there is a subsequence $g_{j_{k}}$ and (not necessarily distinct) points $a, b$ in the circle $\partial \mathbb{S}_{+}$such that

- the sequences $g_{j_{k}}(x) \rightarrow a$ locally uniformly in $\partial \mathbb{S}_{+}-\{b\}$, that is, uniformly in any compact subset of $\partial \mathbb{S}_{+}-\{b\}$, and

- $g_{j_{k}}^{-1}(y) \rightarrow b$ locally uniformly on $\partial \mathbb{S}_{+}-\{a\}$ respectively as $k \rightarrow$ $\infty$.

(See 4] for details.) We remark that

$$
a, b \in \Lambda \text {. }
$$

For later purpose, we say that $a$ is an attractor point and $b$ is a repeller point of the sequence $\left\{g_{j_{k}}\right\}$. Let $a_{i}$ and $r_{i}$ denote the attracting fixed point and the repelling fixed point of $g_{i}$ respectively.

Lemma 4.2. Let $g_{j_{k}}$ be a sequence satisfying the above convergence group properties. Suppose that the attractor point a is distinct from the repeller point $b$. Then it follows that

$$
\left\{a_{j_{k}}\right\} \rightarrow a \text { and }\left\{r_{j_{k}}\right\} \rightarrow b
$$

Proof. One can use the fact that if a continuous map $f$ sends a closed arc $I, I \subset \partial \mathbb{S}_{+}$, to its interior $I^{o}$, then the fixed points are in the image $f(I)$. (See also the $\epsilon$-hyperbolicity in p.256 of [2]. Since $a \neq b$, these transformations are uniformly $\epsilon$-hyperbolic.)

Let $\mathbb{U} \mathbb{S}_{+}$be the unit tangent vector bundle over $\mathbb{S}_{+}$, and let $\mathbb{U} \mathbb{S}_{+} / \Gamma=$ $\mathbb{U} \Sigma_{+}$be the unit tangent vector bundle over $\mathbb{S}_{+} / \Gamma=\Sigma_{+}$. By following the geodesics in $\Sigma_{+}$, we obtain a geodesic flow

$$
\Phi: \mathbb{U} \Sigma_{+} \times \mathbb{R} \rightarrow \mathbb{U} \Sigma_{+} .
$$

A geodesic current is a Borel probability measure on $\mathbb{U} \mathbb{S}_{+} / \Gamma$ invariant under the geodesic flow which is supported on a union of nonwandering geodesics. Let $\mathcal{C}\left(\mathbb{S}_{+} / \Gamma\right)$ denote the space of the space of all geodesic currents with the weak-* topology.

Let $\mathbb{U}_{\text {rec }} \Sigma_{+}$denote the set of unit tangent vectors of nonwandering geodesics on $\Sigma_{+}$. We recall

Lemma 4.3. Let $\Sigma_{+}$be as above. Then

- $\mathbb{U}_{\mathrm{rec}} \Sigma_{+} \subset \mathbb{U} \Sigma_{+}$is a connected, compact, geodesic flow invariant set.

- The inverse image $\mathbb{U}_{\text {rec }} \mathbb{S}_{+}$of $\mathbb{U}_{\mathrm{rec}} \Sigma_{+}$in $\mathbb{U}_{\mathrm{rec}} \mathbb{S}_{+}$is precisely the set of geodesics with both endpoints in $\Lambda$. 
- The set

$$
\begin{aligned}
\left\{(a, r) \in \partial \mathbb{S}_{+} \times \partial \mathbb{S}_{-} \mid\right. & a \text { is an attracting fixed point and } \\
r & \text { is a repelling fixed point of } g \in \Gamma\}
\end{aligned}
$$

is dense in $\Lambda \times \Lambda$.

Proof. The first item is in Lemma 1.2 in [46] and the second item is in the proof of the same lemma. The connectedness is also proved in Lemma 1.3 in [46]. The third item follows from the fact that the set of closed geodesic orbits are dense in $\mathbb{U}_{\text {rec }} \Sigma_{+}$as the flow is Anosov (see [50]).

Let $\Delta(\Lambda)$ denote the diagonal of $\Lambda \times \Lambda$. Such pairs $\left(\lambda_{u}, \lambda_{s}\right) \in$ $\Lambda \times \Lambda-\Delta(\Lambda)$ correspond to nonwandering geodesics in the quotient hyperbolic surface. Thus, the set of closed geodesics is dense in the set of nonwandering geodesics by Lemma 4.3 .

We have a compactification picture of the above phenomena: For a nonelementary Fuchsian group $\Gamma_{0} \subset \operatorname{PGL}(2, \mathbb{R})$, the closure in the projective space $\mathrm{P}\left(\operatorname{Mat}_{2}(\mathbb{R})\right)$ equals $\Gamma_{0} \cup(\Lambda \times \Lambda)$, where $\Lambda \subset \partial \mathbb{S}_{+}$is the limit set of $\Gamma_{0}$. Here we could write

$$
\mathrm{P}\left(\operatorname{Mat}_{2}(\mathbb{R})\right)=\operatorname{PGL}(2, \mathbb{R}) \cup\left(\mathbb{R} P^{1} \times \mathbb{R P}^{1}\right)
$$

where $\mathbb{R} P^{1} \times \mathbb{R} P^{1}$ corresponds to the set of projective equivalence classes of rank-one $2 \times 2$ real matrices. Projective equivalence classes of such matrices are completely determined by their kernels and images, which are arbitrary lines through the origin in $\mathbb{R}^{2}$.

\section{ReAl PRojective SchottKy Uniformizations}

Goldman constructed an $\mathbb{R P}^{2}$-surface $\Sigma$ with a free holonomy group in 42 . The holonomy group is in the image $\operatorname{PSO}(2,1)$ of $\mathbf{S O}(2,1)$ in $\operatorname{PGL}(3, \mathbb{R})$. We will show that a domain in $\mathbf{S}_{\infty}^{2}$ regularly covers $\Sigma$.

Let $\Gamma$ be a free group of rank $\geq 2$ in $\operatorname{Aut}\left(\mathbf{S}^{3}\right)$ acting on $\mathbb{S}_{+} \cup \mathbb{S}_{-}$ properly and freely but without parabolics. We do not require $\Gamma$ to act properly and freely on $E$ in this section. (We will use the notation of $\$ 4.2$ letting $G=\Gamma$. )

5.1. The construction of the $\Gamma$-invariant domain. Recalling $\$ 4.2$, we assume $\mathbb{L}(\Gamma) \subset \mathrm{SO}(2,1)^{\circ}$ initially and so $\Gamma$ acts on $\mathbb{S}_{+}$. Then $\mathbb{S}_{+} / \Gamma$ is a complete genus $\tilde{\mathrm{g}}$ hyperbolic surface with $\mathrm{b}$ ideal boundary components where $\mathrm{b} \geq 1$ and $\tilde{\mathrm{g}}$ is just some integer $\geq 0$. In other words, $\mathbb{S}_{+} / \Gamma$ can be compactified by adding boundary components to a compact surface $\Sigma_{+}^{\prime}$ with b boundary components. The universal cover $\tilde{\Sigma}_{+}^{\prime}$ of $\Sigma_{+}^{\prime}$ is identified with the union of a domain $\mathbb{S}_{+}$and a 
collection of open arcs in $\partial \mathbb{S}_{+}$. (At the moment, we do not require that $\Gamma$ acts properly or freely on $\mathrm{E}$. )

Let $\Lambda$ denote the limit set of an orbit $\Gamma(x)$ for $x \in \mathbb{S}_{+}$. We identify $\tilde{\Sigma}_{+}^{\prime}$ as $\mathrm{Cl}\left(\mathbb{S}_{+}\right)-\Lambda$. Denote the components of $\partial \Sigma^{\prime}$ by $\partial_{i} \Sigma^{\prime}$ where $i=1, \ldots$, b.

$$
\tilde{\Sigma}_{+}^{\prime}=\mathbb{S}_{+} \cup \bigcup_{i \in \mathcal{J}} \partial_{i} \tilde{\Sigma}_{+}^{\prime}
$$

We will be using the same symbols for the group $\Gamma$ in $\operatorname{Isom}^{+}(\mathrm{E})$ its extension to $\mathbf{S}^{3}$ and its element $g$ and its extension to $\mathbf{S}^{3}$. We identify $\mathbf{S}_{\infty}^{2}$ with the boundary of $\mathscr{H}$, called the boundary sphere. $\Gamma$ acts on the standard circles $\partial \mathbb{S}_{ \pm}$and the interiors $\mathbb{S}_{ \pm}$in $\mathbf{S}_{\infty}^{2}$. Then each nonidentity element $g$ of $\Gamma$ has an attracting fixed point $a$ in $\partial \mathbb{S}_{+}$, a repelling fixed point $r$ in $\partial \mathbb{S}_{+}$, and a saddle-type fixed point $s$ in $\mathbb{S}_{0}$. Of course, their antipodes in $\mathbf{S}_{\infty}^{2}$ are also an attracting fixed point $a_{-}$in $\partial \mathbb{S}_{-}$and a repelling fixed point $r_{-}$in $\partial \mathbb{S}_{-}$and a saddle-type fixed point $s_{-}$in $\mathbb{S}_{0}$ respectively.

Since $g$ acts on $\partial \mathbb{S}_{+}$and fixes $a$ and $r$, we obtain that $g$ acts on the two 1-dimensional subspaces tangent to $\partial \mathbb{S}_{+}$at $a$ and $r$. Hence, $\left\{s, s_{-}\right\}$ is their intersection, and also

$$
\left\{s, s_{-}\right\} \subset \varepsilon(a) \cup \varepsilon(r)
$$

as $\varepsilon(a)$ and $\varepsilon(r)$ are disjoint halves of these 1-dimensional subspaces.

We now define $\tilde{\Sigma}_{-}^{\prime}:=\mathscr{A}\left(\tilde{\Sigma}_{+}^{\prime}\right)$. Then $\Gamma$ acts properly and freely on $\tilde{\Sigma}_{-}^{\prime}$ by antipodality, and $\tilde{\Sigma}_{-}^{\prime} / \Gamma$ is a compact $\mathbb{R} P^{2}$-surface with geodesic boundary diffeomorphic to $\Sigma_{+}^{\prime}$. Denote it by $\Sigma_{-}^{\prime}$. Also, let $\Omega_{-}$be the set of antipodal points to $\Omega_{+}$. Then $\Gamma$ acts properly and freely on $\Omega_{+}$ and $\Omega_{-}$. Denote the respective compact quotient surfaces with geodesic boundary by $\Sigma_{+}^{\prime \prime}$ and $\Sigma_{-}^{\prime \prime}$.

For each point $x$ of $\partial \mathbb{S}_{+}$, we recall that $\varepsilon(x)$ is an open segment of length $\pi$ with end points $x$ and $-x$ tangent to $\partial \mathbb{S}_{+}$. The open arcs $\varepsilon(x)$ for $x \in \partial \mathbb{S}_{+}$give leaves of the foliation $\mathcal{F}$ in $\mathbb{S}_{0}$. Let us give an induced orientation on $\Sigma_{+}^{\prime}$ and hence an induced orientation on each $\mathbf{l}_{i}$ for $i \in \mathcal{J}$. Let $p_{i}$ and $q_{i}$ denote the forward and backward end points of $\mathbf{l}_{i}$. We draw a segment $s_{i}=\varepsilon\left(p_{i}\right)$. We also draw a segment $t_{i}=\varepsilon\left(q_{i}\right)$. Then $\mathbf{l}_{i}, s_{i}, t_{i}$, and $\mathbf{l}_{i-}$ bound an open disk $E_{i}$ invariant under $\left\langle\mathbf{g}_{i}\right\rangle$, which we call a strip. We denote by $\mathcal{R}_{i}$ the open strip union with $\mathbf{l}_{i}$ and $\mathbf{l}_{i-}$; that is,

$$
\mathcal{R}_{i}=E_{i} \cup \mathbf{l}_{i} \cup \mathbf{l}_{i-}, \partial E_{i}=\mathbf{l}_{i} \cup s_{i} \cup t_{i} \cup \mathbf{l}_{i-} .
$$

(See Figures 1, 4, and 2 for the pictures of these arcs tangent to $\mathbb{S}_{+}$ and $\mathbb{S}_{-}$.)

Using $\mathcal{F}$, we obtain: 
Proposition 5.1. The strips $\mathcal{R}_{i}$ and $\mathcal{R}_{j}$ are disjoint for $i \neq j$ where $i, j \in \mathcal{J}$.

Proof. Since $s_{i}, t_{i}, s_{j}, t_{j}$ are all leaves of $\mathcal{F}$ corresponding to distinct points of $\partial \mathbb{S}_{+}$, the mutual disjointness of these arcs follows. (See Figure 4.) $s_{i}$ does not intersect $t_{i}, s_{j}, t_{j}$ for $i \neq j$, where $i, j \in \mathcal{J}$, and $t_{i}$ does not intersect $s_{i}, s_{j}, t_{j}$ for $i \neq j$. Furthermore $\mathbf{l}_{i}$ and $\mathbf{l}_{i-}$ for $i \in \mathcal{J}$ are mutually disjoint by (7). Thus the boundary $\partial \mathcal{R}_{i}$ and $\partial \mathcal{R}_{j}$ for $i \neq j$ are disjoint. Hence, the conclusion follows.

Goldman [41] classified compact annuli with geodesic boundary and holonomy matrices that are diagonalizable with distinct positive eigenvalues following Nagano-Yagi [57]. (See [21] also.)

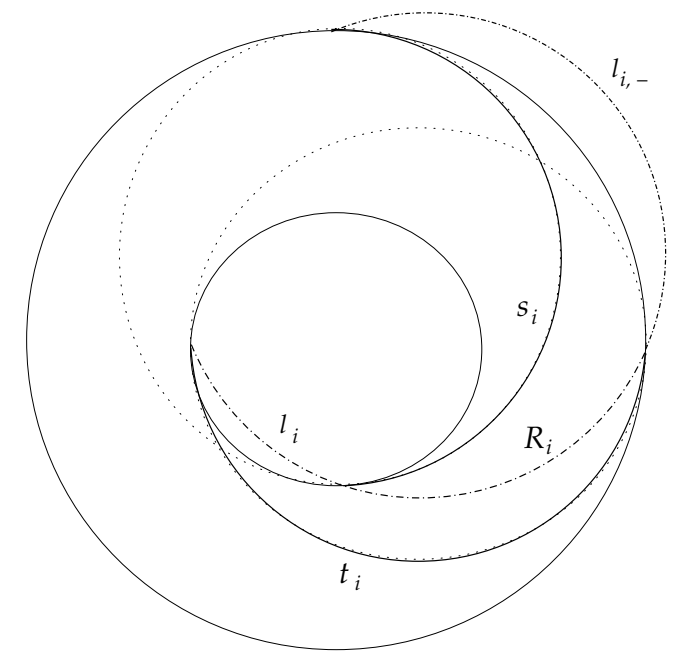

Figure 4. The two stereographically projected "lune" regions which cover $\pi$-annuli.

Proposition 5.2. Given $\mathcal{R}_{i}$ for $i \in \mathcal{J}$ and $\gamma \in \Gamma$, we either have

$$
\gamma\left(\mathcal{R}_{i}\right)=\mathcal{R}_{i} \text { or } \gamma\left(\mathcal{R}_{i}\right) \cap \mathcal{R}_{i}=\emptyset .
$$

In the former case, $\gamma=\mathbf{g}_{i}^{n}, n \in \mathbb{Z}$ for the deck transformation $\mathbf{g}_{i}$ corresponding to $\mathbf{l}_{i}$. Furthermore, $\mathcal{R}_{i} /\left\langle\mathbf{g}_{i}\right\rangle$ is a compact annulus with geodesic boundary that decomposes into two $\pi$-annuli or four elementary annuli along some collection of simple closed geodesics.

Proof. Suppose that $\mathcal{R}_{i} \cap \gamma\left(\mathcal{R}_{i}\right) \neq \emptyset$ holds. Then $\mathbf{l}_{i} \cap \gamma\left(\mathbf{l}_{i}\right) \neq \emptyset$ holds since the $\operatorname{arcs} t_{i}$ are mutually disjoint. Hence, (7) implies the result.

There is a subspace $l$ of dimension 1 , a geodesic circle, containing $\mathbf{l}_{i}$ and $\mathbf{l}_{i-}$ in $\mathbf{S}_{\infty}^{2} . l$ and $t_{i}$ bound a closed disk $D_{1}$ and if we remove $t_{i}$ and the fixed points at the ends of $\mathbf{l}_{i}$, this region covers a $\pi$-annulus with principal boundary components. The similar statement is true for 
the disk bounded by $l$ and $s_{i}$. Therefore, $A_{i}=\mathcal{R}_{i} /\left\langle\mathbf{g}_{i}\right\rangle$ is a union of two compact $\pi$-annuli. In other words, $l-\mathrm{Cl}\left(\mathbf{l}_{i}\right)-\mathrm{Cl}\left(\mathbf{l}_{i-}\right)$ maps to a simple closed principal geodesic decomposing $A_{i}$ into two $\pi$-annuli with principal boundary. (See Figure 4.) Also, each $\pi$-annulus decomposes into two elementary annuli. (See $\$ 4.1$.)

We say that for $i, j \in \mathcal{J}$, the annulus $\mathcal{R}_{i} /\left\langle\mathbf{g}_{i}\right\rangle$ is equivalent to $\mathcal{R}_{j} /\left\langle\mathbf{g}_{j}\right\rangle$ if $\mathcal{R}_{j}=g\left(\mathcal{R}_{i}\right)$ and $g \mathbf{g}_{i} g^{-1}=\mathbf{g}_{j}^{ \pm}$for $g \in \Gamma$. Thus, in fact, there are only b equivalence classes of annuli of above form.

We define

$$
\mathcal{A}_{i}:=\mathcal{R}_{i} \cap \mathbb{S}_{0}=\bigcup_{x \in \partial_{i} \mathbb{S}_{+}} \varepsilon(x) \text { for } i \in \mathcal{J}
$$

We note that $\mathcal{A}_{i} \subset \mathcal{R}_{i}$ for each $i \in \mathcal{J}$. We finally define an open domain in $\mathbf{S}_{\infty}^{2}$ :

$$
\begin{aligned}
\tilde{\Sigma} & =\tilde{\Sigma}_{+}^{\prime} \cup \coprod_{i \in \mathcal{J}} \mathcal{R}_{i} \cup \tilde{\Sigma}_{-}^{\prime} \\
& =\tilde{\Sigma}_{+}^{\prime} \cup \coprod_{i \in \mathcal{J}} \mathcal{A}_{i} \cup \tilde{\Sigma}_{-}^{\prime} \\
& =\Omega_{+} \cup \coprod_{i \in \mathcal{J}} \mathcal{R}_{i} \cup \Omega_{-} \\
& =\mathbf{S}_{\infty}^{2}-\bigcup_{x \in \Lambda} \operatorname{Cl}(\varepsilon(x)) .
\end{aligned}
$$

Since the collection whose elements are of form $\mathcal{R}_{i}$ mapped to itself by $\Gamma$, we showed that $\Gamma$ acts on this open domain.

5.2. The $\mathbb{R P}^{2}$-surface. First, $\tilde{\Sigma}$ does not contain any fixed point: Suppose that $g \in \Gamma$ acts on $\mathbf{l}_{i}$ for some $i \in \mathcal{J}$. Then $g$ acts on $\mathcal{R}_{i}$ and the four attracting and repelling fixed points are the vertices of $\mathcal{R}_{i}$ and two saddle-type fixed points are in $s_{i}$ or $t_{i}$. Hence, they are outside $\tilde{\Sigma}$.

Suppose that $g \in \Gamma-\{\mathrm{I}\}$ does not act on any of the boundary components. Then $g$ has four attracting and repelling fixed points $a, r \in \partial \mathbb{S}_{+}$and $a_{-}, r_{-} \in \partial \mathbb{S}_{-}$. By $(10)$, saddle-type fixed points $s$ and $s_{-}$are either on a great segment $\varepsilon(a)$ or the other one $\varepsilon(r)$ tangent to $\partial \mathbb{S}_{+}$at $a$ and $r$ respectively. Since $a, r \in \Lambda$ holds, the fixed points are outside $\tilde{\Sigma}$ by $(12)$.

Let $G \subset \mathrm{SO}(2,1)^{\circ}$ be a subgroup whose elements preserve orientation on $\mathbb{S}_{+}$, and any open domain $\mathcal{D}$ an open domain on $\mathbf{S}_{\infty}^{2}$ upon which where $G$ acts freely and properly. Then $\mathcal{D} / G$ has an induced orientation from $\mathbb{S}_{+}$.

Theorem 5.3. Let $\Gamma$ be an orientation-preserving finitely generated group in $\mathrm{SO}(2,1)^{\circ}$ without parabolics acting freely and properly on $\mathbb{S}_{+}$ 
isomorphic to a free group of finite rank $\geq 2$. Denote the ideal boundary components of $\mathbb{S}_{+} / \Gamma$ by $\partial_{i}\left(\mathbb{S}_{+} / \Gamma\right)$, for $i=1, \ldots$, b. Then

- $\Gamma$ acts properly and freely on an open domain $\mathcal{D}$ in $\mathbf{S}_{\infty}^{2}$ and $\mathcal{D} / \Gamma$ is homeomorphic to a closed surface of genus $\mathrm{g}, \mathrm{g} \geq 2$.

- As an $\mathbb{R P}^{2}$-surface $\mathcal{D} / \Gamma$ decomposes along simple closed principal geodesics into a union of $\mathrm{b}$ annuli $A_{1}, \ldots, A_{\mathrm{b}}$ and two convex $\mathbb{R P}^{2}$-surfaces $\Sigma_{+}^{\prime \prime}$ and $\Sigma_{-}^{\prime \prime}$. Each annulus $A_{i}, i=1, \ldots, \mathrm{b}$ decomposes into two $\pi$-annuli.

- $\Gamma$ also acts properly and freely on $\mathcal{D}_{-}:=\mathscr{A}(\mathcal{D})$, and $\mathcal{D}_{-} / \Gamma$ is a closed $\mathbb{R P}^{2}$-surface diffeomorphic to $\mathcal{D} / \Gamma$.

- The antipodal map $\mathscr{A}: \mathbf{S}_{\infty}^{2} \rightarrow \mathbf{S}_{\infty}^{2}$ induces a projective diffeomorphism between $\mathcal{D} / \Gamma$ and $\mathcal{D}_{-} / \Gamma$.

Proof. We begin by letting $\mathcal{D}=\tilde{\Sigma}$ obtained as above in (11). We showed that $\Gamma$ has no fixed point on $\mathcal{D}$. Let $\mathrm{Cl}_{\mathcal{D}}\left(\mathcal{A}_{i}\right)$ denote the closure of $\mathcal{A}_{i}$ in $\mathcal{D}$ for each $i \in \mathcal{J}$.

Now $\Gamma$ acts properly on $\tilde{\Sigma}_{+}^{\prime}$ and $\tilde{\Sigma}_{-}^{\prime}$ and each $g \in \Gamma$ sends $\operatorname{Cl}_{\mathcal{D}}\left(\mathcal{A}_{i}\right)$ to $\mathrm{Cl}_{\mathcal{D}}\left(\mathcal{A}_{j}\right)$ for $j \neq i$ or $g$ is in the infinite cyclic subgroup of $\Gamma$ acting

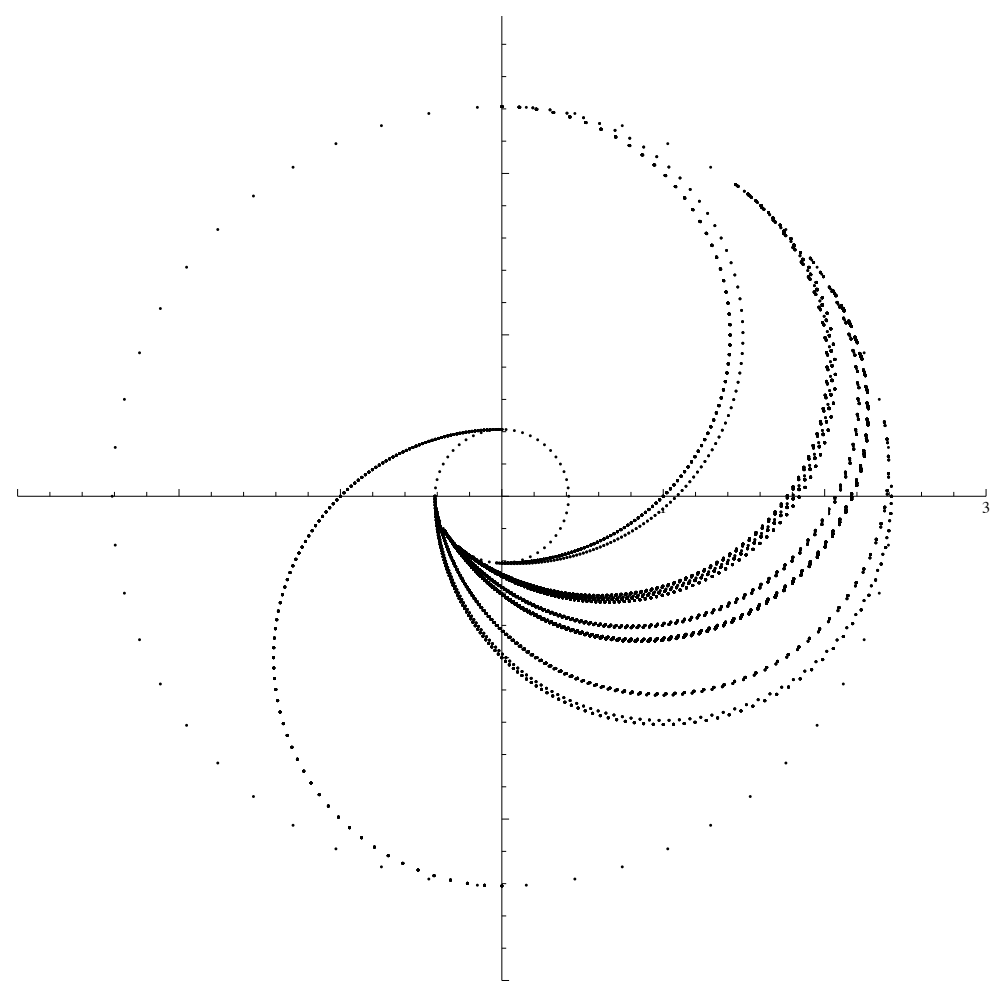

FiguRE 5. A figure representing an actual free group action with some of the arcs $s_{i}$ and $t_{i}$ drawn in point-plots on the stereographically projected sphere as in Figure 2. 
properly on $\mathrm{Cl}_{\mathcal{D}}\left(\mathcal{A}_{i}\right)$ if $g\left(\mathrm{Cl}_{\mathcal{D}}\left(\mathcal{A}_{i}\right)\right)=\mathrm{Cl}_{\mathcal{D}}\left(\mathcal{A}_{i}\right)$. Hence, $\Gamma$ acts properly on $\bigcup_{i \in \mathcal{J}} \mathrm{Cl}_{\mathcal{D}}\left(\mathcal{A}_{i}\right)$.

For any compact set $K \subset \tilde{\Sigma}$, the compact sets

$$
\begin{aligned}
K_{1} & :=K \cap \tilde{\Sigma}_{+}^{\prime}, \\
K_{-1} & :=K \cap \tilde{\Sigma}_{-}^{\prime}, \\
K_{0} & :=K \cap \bigcup_{i \in \mathcal{J}} \operatorname{Cl}_{\mathcal{D}}\left(\mathcal{A}_{i}\right)
\end{aligned}
$$

satisfy:

$$
K=K_{+} \cup K_{-} \cup K_{0}
$$

Since each of $\mathrm{Cl}_{\mathcal{D}}\left(\mathcal{A}_{i}\right)$ has an open neighborhood $O_{j}$ forming a mutually disjoint collection of open sets covering $K_{0}$, it follows that $K_{0}$ meets only finitely many $\mathrm{Cl}_{\mathcal{D}}\left(\mathcal{A}_{i}\right)$.

If $g(K) \cap K \neq \emptyset$, we have $g\left(K_{i}\right) \cap K_{i} \neq \emptyset$ for some $i=-1,0,1$. Since $\Gamma$ acts properly on $\tilde{\Sigma}_{+}^{\prime}$ and $\tilde{\Sigma}_{-}^{\prime}$ and $\bigcup_{i \in \mathcal{J}} \mathrm{Cl}_{\mathcal{D}}\left(\mathcal{A}_{i}\right)$ respectively, only finitely many elements $g$ of $\Gamma$ satisfy $g\left(K_{i}\right) \cap K_{i} \neq \emptyset$ for each $i$, $i=-1,0,1$. We showed that $\Gamma$ acts properly and freely on $\mathcal{D}$.

Since $\tilde{\Sigma}_{+}^{\prime} / \Gamma$ and $\tilde{\Sigma}_{-}^{\prime} / \Gamma$ are compact surfaces, and $\bigcup_{i \in \mathcal{J}} \mathrm{Cl}_{\mathcal{D}}\left(\mathcal{A}_{i}\right) / \Gamma$ is a union of finitely many compact annuli, $\mathcal{D} / \Gamma$ is a closed surface.

For the second item, $\mathcal{D} / \Gamma$ decomposes along the images of the union of $\mathbf{l}_{i}, \mathbf{l}_{i-}, i \in \mathcal{J}$ to $\Sigma_{+}^{\prime \prime}$ and $\Sigma_{-}^{\prime \prime}$ and annuli with boundary components that are images of the union of $\mathbf{l}_{i}$ and $\mathbf{l}_{i-}, i \in \mathcal{J}$. Proposition 5.2 implies that these annuli are obtained from two $\pi$-annuli.

Since $\mathscr{A}\left(\Gamma \mid \mathbf{S}_{\infty}^{2}\right) \mathscr{A}^{-1}=\Gamma \mid \mathbf{S}_{\infty}^{2}$ and $\mathscr{A}(\mathcal{D})=\mathcal{D}_{-}$hold, $\Gamma$ acts properly and freely on $\mathcal{D}_{-}$. Let $\kappa$ denote the induced diffeomorphism $\Sigma \rightarrow \mathcal{D}_{-} / \Gamma$ where $\kappa$ sends $\Sigma_{-}^{\prime \prime}$ to $\Sigma_{+}^{\prime \prime}$ and vice versa. $\kappa$ sends annuli in $\Sigma$ to ones in $\mathcal{D}_{-} / \Gamma$ but they do not share subdomains in the universal cover. (Actually $\mathcal{R}_{i}$ goes to its antipodal image which is distinct from $\mathcal{R}_{i}$ for each $i \in \mathcal{J}$.)

The rest follows from this observation.

The proof of Theorem 1.1. If $\mathbb{L}(\Gamma) \subset \mathrm{SO}(2,1)^{\circ}$, then Theorem 5.3 proves Theorem 1.1. Now, we consider the case when $\mathbb{L}(\Gamma)$ is a subgroup of SO $(2,1)$ in general. Define $\Gamma^{\prime}$ to be the subgroup of $\Gamma$ of index 2 so that $\mathbb{L}\left(\Gamma^{\prime}\right) \subset \mathrm{SO}(2,1)^{o}$ using above notations.

Let $\psi$ be the element of $\Gamma-\Gamma^{\prime}$. Then $\psi$ is orientation-preserving but $\psi\left(\mathbb{S}_{+}\right)=\mathbb{S}_{-}$. Let $\mathcal{D}^{\prime}$ be defined by (11) for $\Gamma^{\prime}$. Since $\psi$ preserves orientation, $\psi$ acts on $\mathcal{D}^{\prime}$. Thus, $\psi$ acts on $\mathcal{D}^{\prime}$ and hence $\Gamma$ acts on $\mathcal{D}^{\prime}$.

Recall from the introduction that $\mathbb{L}^{\prime}(\Gamma)$ is a free group of rank $\geq 2$ acting freely on $\mathbb{S}_{+}$as a subgroup of $\operatorname{PGL}(3, \mathbb{R})$ and $\mathbb{S}_{+} / \mathbb{L}^{\prime}(\Gamma)$ is a nonorientable complete hyperbolic surface. Since $\mathbb{L}^{\prime}(\Gamma)$ acts freely on $\mathbb{S}_{+}, \psi$ goes to an element of $\operatorname{PGL}(3, \mathbb{R})$ that acts freely on $\mathbb{S}_{+}$and on 
$\bigcup_{i \in \mathcal{J}} \partial_{i} \mathbb{S}_{+}$. Hence, $\psi$ as an element of $\operatorname{Aut}\left(\mathbf{S}^{3}\right)$ acts without fixed point on

$$
\bigcup_{i \in \mathcal{J}} \bigcup_{x \in \partial_{i} \mathbb{S}_{+}} \varepsilon(x)=\coprod_{i \in \mathcal{J}} \mathcal{A}_{i}
$$

since $\psi$ does not act on any fiber of the fibration. Hence $\psi$ has no fixed point on

$$
\tilde{\Sigma}_{+}^{\prime} \cup \coprod_{i \in \mathcal{J}} \mathcal{A}_{i} \cup \tilde{\Sigma}_{-}^{\prime}
$$

$\mathcal{D}^{\prime} / \Gamma^{\prime}$ double-covers a closed surface $\mathcal{D} / \Gamma$.

Finally, it is straightforward to verify the same statements for $\mathscr{A}(\mathcal{D})$. Replacing with this set is equivalent to replacing $\mathrm{Cl}(\varepsilon(x))$ with $\mathrm{Cl}\left(\varepsilon(x)_{-}\right)$ for every appropriate point $x$ in (12).

We prove the uniqueness under the assumption that $\Sigma$ has a regular covering domain $\mathcal{D}^{\prime} \subset \mathrm{S}_{\infty}^{2}$. Theorem $\mathrm{R}$ of 42 implies that for a given Fuchsian holonomy group $\Gamma$, a closed $\mathbb{R P}^{2}$-surface $\Sigma$ is obtained as follows: Let $\Sigma^{\prime}$ denote a closed properly convex $\mathbb{R} P^{2}$-surface covered by $\mathbb{S}_{+}$. We cut along the union of mutually disjoint simple closed geodesics in $\Sigma^{\prime}$, and insert $\mathbb{R P}^{2}$-annuli with principal geodesic boundary with conjugate holonomy to the geodesics by projectively pasting with the boundary components of the split surface. (See [62] and [21] for details.) This construction is called grafting. Such an annulus is a union of elementary annuli meeting in geodesic boundary components.

By Proposition 4.5 of [21], each boundary component of the inserted annulus is principal as we used simple closed geodesics in a properly convex closed $\mathbb{R P}^{2}$-surface $\Sigma^{\prime}$. Each inserted annulus has a domain $K$ in $\mathbf{S}_{\infty}^{2}$ covering it since $\mathcal{D}^{\prime}$ is a domain in $\mathbf{S}_{\infty}^{2}$. The removed simple closed geodesics correspond to $\mathbf{l}_{i}$ and $\mathbf{l}_{i-}$ for $i \in \mathcal{J}$. $K$ must have boundary $\operatorname{arcs} \mathbf{l}_{i}$ and $\mathbf{l}_{i-}$ for some $i \in \mathcal{J}$. Since $K-\mathbb{S}_{+}-\mathbb{S}_{-}$is a domain in $\mathbf{S}_{\infty}^{2}$ and covers the compact annulus with boundary, $K-\mathbb{S}_{+}-\mathbb{S}_{-}$is a union of components of

$$
\mathbf{S}_{\infty}^{2}-\left(\mathbb{S}_{+} \cup \mathbb{S}_{-}\right)-\left(l_{1} \cup l_{2} \cup l_{3}\right)
$$

and components of

$$
\left(l_{1} \cup l_{2} \cup l_{3}\right)-\left\{a, a_{-}, r, r_{-}, s, s_{-}\right\}
$$

using the notation of $\vartheta^{\prime}$ of Section 4.1 adopted to $\mathbf{g}_{i}$. By Goldman [41], we can show that $K=\mathcal{R}_{i}$ or $K=\mathcal{R}_{i-}$ for some $i \in \mathcal{J}$. (See also 62].) In order for these domains to be disjoint, they are all of from $\mathcal{R}_{i}$ or all of form $\mathcal{R}_{i-}$ for $i \in \mathcal{J}$. Now, it follows that $\mathcal{D}$ or $\mathcal{D}_{-}$are only possible domains covering closed $\mathbb{R P}^{2}$-surfaces with the holonomy groups equal to $\mathbb{L}(\Gamma)$. (See also [24].) 


\section{6. $\Gamma$ ACTS PROPERLY ON E $\cup \tilde{\Sigma}$.}

First we briefly summarize the relevant results of Goldman, Labourie, and Margulis in [46] and [45]. We will need some parts of this section for our main proof.

6.1. Diffused Margulis invariants. We assume that $\Gamma$ acts freely and properly on $\mathbf{E}$ and hence on $\mathbb{S}_{+}$(or equivalent on $\left.\mathbb{S}_{-}\right)$. $\Gamma$ acts on $\mathbb{S}_{+}$as a geometrically finite Fuchsian group.

If $\Gamma$ acts freely and properly on $E$, then Margulis invariants of nonidentity elements are all positive or all negative by the opposite sign lemma. (See [46].) By choosing the opposite orientation of $\mathrm{E}$, we can change the all signs of the invariants. We henceforth assume that the Margulis invariants of nonidentity elements are all positive.

The Margulis invariant $\mu(c)$ of a current $c$ supported on a simple closed geodesic $\hat{c}$ on $\mathbb{S}_{+} / \Gamma$ is given by $\mu(g) / l_{\mathbb{S}_{+}}(\hat{c})$ for the corresponding element $g \in \Gamma$ and the hyperbolic length $l_{\mathbb{S}_{+}}(\hat{c})$. In the second main theorem of [46], they define the diffused Margulis invariants on $\mathcal{C}\left(\mathbb{S}_{+} / \Gamma\right)$ by continuously extending the Margulis invariants.

These invariants of elements of $\mathcal{C}\left(\mathbb{S}_{+} / \Gamma\right)$ are positive by the second main theorem in [46]. The subspace of currents supported on closed geodesics with total measure 1 is precompact in $\mathcal{C}\left(\mathbb{S}_{+} / \Gamma\right)$. Thus, there exists $C>0$ such that

$$
1 / C \leq \frac{\mu(g)}{l_{\mathbb{S}_{+}}(g)} \leq C \text { for } g \in \Gamma-\{\mathrm{I}\}
$$

for all $g \in \Gamma$, where $l_{\mathbb{S}_{+}}(g)$ denotes the minimum of the hyperbolic distances between $x$ and $g(x)$ for $x \in \mathbb{S}_{+}$.

6.2. Neutralized sections. We construct a flat affine bundle $\mathbf{E}$ over the unit tangent bundle $\mathbb{U} \Sigma_{+}$of $\Sigma_{+}$by taking the quotient of $E \times \mathbb{U S} \mathbb{S}_{+}$ by the diagonal action

$$
\gamma(v, x)=(\gamma(v), D \gamma(x)), v \in \mathrm{E}, x \in \mathbb{U S}_{+}, \gamma \in \Gamma
$$

where $D \gamma$ is the differential map. The cover of $\mathbf{E}$ is denoted by $\hat{\mathbf{E}}$ and is identical with $\mathrm{E} \times \mathbb{U} \mathbb{S}_{+}$. We denote by

$$
\pi_{\mathrm{E}}: \hat{\mathbf{E}}=\mathrm{E} \times \mathbb{U} \mathbb{S}_{+} \rightarrow \mathrm{E}
$$

the projection.

We define $\mathbf{V}$ as the quotient space of $\mathbf{V} \times \mathbb{U} \mathbb{S}_{+}$under the diagonal action

$$
\gamma(v, x)=(\mathbb{L}(\gamma)(v), D \gamma(x)), v \in \mathrm{V}, x \in \mathbb{U S}_{+}, \gamma \in \Gamma
$$

Given $\mathrm{u} \in \mathbb{U S}_{+}$, let $l(\mathrm{u})$ denote the geodesic tangent to $\mathrm{u}$ in the hyperbolic plane $\mathbb{S}_{+}$and $\partial_{+} l(\mathbf{u}) \in \partial \mathbb{S}_{+}$the starting point and $\partial_{-} l(\mathbf{u}) \in$ 
$\partial \mathbb{S}_{+}$the endpoint of $l(\mathbf{u})$. The maps $\mathbb{U} \mathbb{S}_{+} \rightarrow \partial \mathbb{S}_{+}$given by $\mathbf{u} \rightarrow \partial_{ \pm} l(\mathbf{u})$ are smooth maps. We define

$$
\tilde{\boldsymbol{\nu}}: \mathbf{u} \in \mathbb{U} \mathbb{S}_{+} \mapsto\left(\mathrm{u}, \frac{\rho(\mathrm{u}) \times \alpha(\mathrm{u})}{\|\rho(\mathrm{u}) \times \alpha(\mathrm{u})\| \|}\right)
$$

for the null vectors $\rho(\mathbf{u})$ and $\alpha(\mathbf{u})$ where $\||| \cdot||$ is the absolute value of the Lorentzian norm and

$$
((\rho(\mathbf{u})))=\partial_{+} l(\mathbf{u}) \in \partial \mathbb{S}_{+} \text {and }((\alpha(\mathbf{u})))=\partial_{-} l(\mathbf{u}) \in \partial \mathbb{S}_{+}
$$

hold. Since

$$
\mathbb{L}(\gamma)(\tilde{\boldsymbol{\nu}}(x))=\tilde{\boldsymbol{\nu}}(D \gamma(x)), x \in \mathbb{U S}_{+}, \gamma \in \Gamma,
$$

$\tilde{\boldsymbol{\nu}}$ induces a neutral section

$$
\nu: \mathbb{U} \Sigma_{+} \rightarrow \mathbf{V}
$$

(See $§ 4.2$ of [46] for details.)

Let $\nabla$ be the flat connection on $\mathbf{E}$ as a bundle over $\mathbb{U} \mathbb{S}_{+}$with fiber isomorphic to $\mathbf{E}$ induced from the product structure of $\hat{\mathbf{E}}=\mathbf{E} \times \mathbb{U S}_{+}$. (See $\S 3.2$ and 3.3 of [46].) Lemma 8.4 of [46] finds a neutralized section $\mathcal{N}: \mathbb{U}_{\text {rec }} \Sigma_{+} \rightarrow$ E satisfying

$$
\nabla_{X} \mathcal{N}=f \nu
$$

where $X$ is the vector field of geodesic flow on $\mathbb{U}_{\text {rec }} \Sigma_{+}$and $f$ is a positive valued function defined on $\mathbb{U}_{\text {rec }} \Sigma_{+}$.

6.3. Lifting the neutralized section to the coverings. Let $\mathbb{U}_{\text {rec }} \mathbb{S}_{+}$ denote the inverse image of $\mathbb{U}_{\text {rec }} \Sigma_{+}$in $\mathbb{U S}_{+}$. Thus, we find the section $\tilde{\mathcal{N}}: \mathbb{U}_{\text {rec }} \mathbb{S}_{+} \rightarrow \hat{\mathbf{E}}$ lifting $\mathcal{N}$ satisfying

$$
\tilde{\mathcal{N}} \circ \gamma=\gamma \circ \tilde{\mathcal{N}}, \gamma \in \Gamma
$$

Equation (14) also lifts to

$$
\nabla_{X} \tilde{\mathcal{N}}=\tilde{f} \tilde{\boldsymbol{\nu}}
$$

where $X$ is the unit-vector field of geodesic flow on $\mathbb{U S}_{+}, \nabla$ is the flat connection on $\mathbf{E} \times \mathbb{U S}_{+}$, and $\tilde{f}$ is a positive-valued function.

6.4. Mapping the convex core. Let UE denote the space of unit spacelike vectors at each point of $E$ and $\mathbb{U E} / \Gamma$ the space of unit spacelike vectors at each point of $E / \Gamma$. Denote by $\mathbb{U}_{\text {rec }} E$ in $\mathbb{U E}$ the inverse image of the subset of $\mathbb{U}_{\text {rec }} E / \Gamma$ composed of unit tangent spacelike vectors tangent to nonwandering spacelike geodesics.

We give the topology of the space $\mathcal{G}_{\mathrm{rec}} \mathbb{S}_{+}$of oriented geodesics in $\mathbb{S}_{+}$mapping to nonwandering oriented geodesics in $\Sigma_{+}$by the quotient topology from $\mathbb{U}_{\text {rec }} \mathbb{S}_{+}$. Similarly, we give the topology of the space $\mathcal{G}_{\text {rec }} E$ 
of spacelike oriented geodesics in $\mathrm{E}$ mapping to nonwandering geodesics in $E / \Gamma$ by the quotient topology from $\mathbb{U}_{\text {rec }} E$.

A bounded subset of $\mathcal{G}_{\text {rec }} \mathbb{S}_{+}$is a set of geodesics passing a bounded set in $\mathbb{U S}_{+}$, and a bounded subset of $\mathcal{G}_{\text {rec }} \mathrm{E}$ is a set of spacelike geodesics passing a bounded set in UE under the Euclidean metric $d_{E}$.

Proposition 6.1 (Theorem 1 of [45]). The map $\tilde{\mathcal{N}}$ induces a continuous function

$$
\mathcal{G}_{\mathrm{rec}} \mathbb{S}_{+} \stackrel{\mathscr{N}}{\longrightarrow} \mathcal{G}_{\mathrm{rec}} \mathrm{E}
$$

where a bounded set of elements of $\mathcal{G}_{\text {rec }} \mathbb{S}_{+}$maps to a bounded set in $\mathcal{G}_{\text {rec }} \mathrm{E}$.

Proof. $\pi_{\mathrm{E}} \circ \tilde{\mathcal{N}}$ maps a flow segment in $\mathbb{U}_{\text {rec }} \mathbb{S}_{+}$to a spacelike line segment in E. For a flow segment in $\mathbb{U}_{\text {rec }} \mathbb{S}_{+}, \pi_{\mathrm{E}} \circ \tilde{\mathcal{N}}$ sends it to a spacelike line segment in E. Since the geodesic flow transfers to the geodesic flow on E, this induces $\mathscr{N}$.

The next statement follows by the fact that $\mathscr{N}$ is induced by the continuous map $\pi_{\mathrm{E}} \circ \tilde{\mathcal{N}}$ which sends a compact set to a compact set.

6.5. The proof of proper discontinuity. Let $\Gamma$ be as above. We assume initially that $\mathbb{L}(\Gamma) \subset \mathrm{SO}(2,1)^{\circ}$ and $\mathbb{S}_{+} / \Gamma$ is a complete genus $\tilde{\mathrm{g}}$ hyperbolic surface with $\mathrm{b}$ ideal boundary components. As above, we assume that the Margulis invariants of nonidentity elements are all positive.

By Theorem 5.3 ,

$$
\tilde{\Sigma}:=\mathbb{S}_{+} \cup \coprod_{i \in \mathcal{J}} \mathcal{R}_{i} \cup \mathbb{S}_{-} \subset \mathbf{S}_{\infty}^{2}
$$

is a $\Gamma$-invariant open domain. The quotient map $\tilde{\Sigma} \longrightarrow \Sigma$ is a covering space onto a closed $\mathbb{R P}^{2}$-surface $\Sigma$. This surface decomposes as a union of $\Sigma_{+}^{\prime}$ and $\Sigma_{-}^{\prime}$ and annuli $A_{i}$ for $i=1, \ldots$, b.

Proposition 6.2. Let $\Gamma$ be as above. Assume $\mathbb{L}(\Gamma) \subset \mathrm{SO}(2,1)^{\circ}$. Then $\Gamma$ acts freely and properly on $\mathrm{E} \cup \tilde{\Sigma}$.

Proof. Since $\Gamma$ acts freely on both $\mathbb{R}^{3}=\mathrm{E}$ and on $\tilde{\Sigma}$, it acts freely on the union. Since $\Gamma$ is discrete, it suffices to show that the action of $\Gamma$ on $\mathrm{E} \cup \tilde{\Sigma}$ is proper.

Suppose that $\Gamma$ contains a sequence $g_{n}$ and that $K \subset \mathrm{E} \cup \tilde{\Sigma}$ is compact so that

$$
g_{n}(K) \cap K \neq \emptyset
$$

for all $n$. We show that the sequence $g_{n}$ is finite. 
We prove by contradiction: Suppose that $\left\{g_{n}\right\}$ is an infinite sequence of mutually distinct isometries.

Recall that $\Gamma$ acts on $\partial \mathbb{S}_{+}$as a convergence group. Let $a_{n}$ and $r_{n}$ denote the attracting fixed point and the repelling fixed point of $g_{n}$ respectively. We can choose a subsequence $g_{n_{k}}$ satisfying the convergence group property (see \$4.3). For the attracting fixed point $a_{n_{k}}$ and the repelling fixed point $r_{n_{k}}$ in $\partial \mathbb{S}_{+}$of $g_{n_{k}}$, Lemma 4.2 implies:

$$
\begin{aligned}
& \left\{a_{n_{k}}\right\} \rightarrow a \\
& \left\{r_{n_{k}}\right\} \rightarrow b
\end{aligned}
$$

for $a, b \in \partial \mathbb{S}_{+}$provided $a \neq b$.

First, we will consider the case when $a \neq b$ :

6.6. Convergences of the axes. Now letting $\left\{g_{n}\right\}$ denote the subsequence, we note that each $g_{n}$ has an attracting fixed point $a_{n}$ and a repelling fixed point $r_{n}$ in $\partial \mathbb{S}_{+}$. By our conditions, $\left\{a_{n}\right\} \rightarrow a$ and $\left\{r_{n}\right\} \rightarrow b$ hold by Lemma 4.2 .

We define

$$
\nu:=\frac{\beta \times \alpha}{\|\beta \times \alpha\|}
$$

of nonzero vectors $\alpha$ and $\beta$ corresponding to $a$ and $b$ respectively. A Lorentzian isometry element $g_{n}$ acts as a translation on a unique spacelike line $\operatorname{Axis}\left(g_{n}\right)$ in the direction $\nu_{n}$ of eigenvalue 1. Let $\alpha_{n}$ and $\rho_{n}$ denote the null vectors in the directions of $a_{n}$ and $r_{n}$ respectively so that $\alpha_{n} \rightarrow \alpha$ and $\rho_{n} \rightarrow \beta$ hold. (We can assume without loss of generality that

$$
\left\|\left|\rho_{n} \times \alpha_{n}\right|\right\|=1,\||\beta \times \alpha \||=1 .)
$$

We let $\nu_{n}$ be the cross-product of $\rho_{n}$ and $\alpha_{n}$; that is, $\nu_{n}=\mathrm{v}_{0}\left(g_{n}\right)$. Thus $\left(\left(\nu_{n}\right)\right) \rightarrow((\nu)) \in \mathbf{S}_{\infty}^{2}$. Since $\left\{a_{n}\right\} \rightarrow a$, the sequence

$$
\overline{a_{n}\left(\left(\nu_{n}\right)\right) a_{n,-}}=\operatorname{Cl}\left(\varepsilon\left(a_{n}\right)\right)
$$

converges to a segment

$$
\overline{a((\nu)) a_{-}}=\mathrm{Cl}(\varepsilon(a)) .
$$

Since the geodesics with endpoints $a_{n}, r_{n}$ pass the bounded part $K^{\prime \prime}$ of the unit tangent bundle of $\mathbb{S}_{+}$,

$$
\mathrm{Cl}\left(\operatorname{Axis}\left(g_{n}\right)\right)=\mathrm{Cl}\left(\mathscr{N}\left({\overrightarrow{r_{n} a_{n}^{o}}}^{o}\right) \longrightarrow \mathrm{Cl}\left(\mathscr{N}\left(\overrightarrow{b a}^{o}\right)\right)\right.
$$

by the continuity of $\mathscr{N}$ of Proposition 6.1. Hence, each Axis $\left(g_{n}\right)$ passes a point $p_{n}$, and $\left\{p_{n}\right\}$ forms a convergent sequence in $\mathrm{E}$. By choosing a subsequence, we assume without loss of generality that $p_{n} \rightarrow p_{\infty}$ for $p_{\infty} \in \mathrm{E}$. 
Recall that the span of $\operatorname{Axis}\left(g_{n}\right)$ and $r_{n}$ is

$$
S_{n}:=\mathscr{W}^{w s}\left(g_{n}\right) \text {. }
$$

To conclude (I)(i), we obtained a sequence $\left\{g_{n}\right\}$ satisfying the properties:

$$
\begin{aligned}
& \alpha_{n} \rightarrow \alpha, \rho_{n} \rightarrow \beta, \nu_{n} \rightarrow \nu, \\
& a_{n} \rightarrow a, r_{n} \rightarrow b,\left(\left(\nu_{n}\right)\right) \rightarrow((\nu)), p_{n} \rightarrow p_{\infty}, \\
& \mathrm{Cl}\left(\operatorname{Axis}\left(g_{n}\right)\right) \rightarrow \overline{((\nu)) p_{\infty}((-\nu))}, \\
& S_{n} \rightarrow S_{\infty} .
\end{aligned}
$$

The last property follows easily.

6.7. Conjugating to a standard boost form. Next we find a uniformly bounded sequence $h_{n}$ of coordinate changes so that $h_{n} g_{n} h_{n}^{-1}$ is in standard form (4). (See \$3.6.1.) The uniform bounds on $h_{n}$ follow from dynamical properties established in [45] as stated by equation (19).

We now introduce $h_{n} \in \operatorname{Aut}\left(\mathbf{S}^{3}\right)$ used for coordinatizing $\mathbf{S}^{3}$ for each $n$. We choose $h_{n}$ so that

$$
h_{n}\left(a_{n}\right)=\mathrm{e}_{1}, h_{n}\left(\left(\left(\nu_{n}\right)\right)\right)=\mathrm{e}_{2}, h_{n}\left(b_{n}\right)=\mathrm{e}_{3}, h_{n}\left(p_{n}\right)=\mathrm{e}_{4},
$$

and $\operatorname{Axis}\left(g_{n}\right)$ is sent to

$$
\mathrm{e}_{2} \mathrm{e}_{4} \mathrm{e}_{2-}{ }^{\circ} .
$$

By (19) and Lemma 2.1. $\left\{h_{n}\right\}$ can be chosen so that $\left\{h_{n}\right\}$ converges to a bi-Lipschitz map $h \in \operatorname{Aut}\left(\mathbf{S}^{3}\right)$, uniformly in the $C^{s}$-sense for any integer $s \geq 0$.

We can consider $\mathrm{v}_{0}\left(g_{n}\right)=\nu_{n}$ as a tangent vector at the origin $O$ in E. Since $h_{n}$ acts on $\mathrm{E}, h_{n}$ is an affine transformation and hence the linearization $\mathbb{L}\left(h_{n}\right)$ equals the differential $D h_{n, x}$ at any $x \in \mathrm{E}$. By 20 , $\mathbb{L}\left(h_{n}\right)$ sends the unit Lorentzian norm vector $\mathrm{v}_{0}\left(g_{n}\right)$ to a vector parallel to $(0,1,0) \in \mathrm{V}$. By post-composing with a projective map $\Phi_{n}$ fixing $\mathrm{e}_{n}, i=1, \ldots, 4$, we further modify $h_{n}$ so that

$$
D h_{n, p_{n}}\left(\nu_{n}\right)=(0,1,0)_{\mathbf{e}_{4}} \text { and hence } \mathbb{L}\left(h_{n}\right)\left(\mathrm{v}_{0}\left(g_{n}\right)\right)=(0,1,0) \text {. }
$$

Since $\left\{\mathrm{v}_{0}\left(g_{n}\right)\right\}$ is a convergent sequence of vectors, we proved

Lemma 6.3. We can choose the sequences $h_{n}$ satisfying equation (20) convergent uniformly in $C^{s}$ to $h \in \operatorname{Aut}\left(\mathbf{S}^{3}\right)$ for any integer $s \geq 0$ so that

$$
\begin{aligned}
& C^{-1} \mathbf{d}\left(h_{n}(x), h_{n}(y)\right) \leq \mathbf{d}(x, y) \leq C \mathbf{d}\left(h_{n}(x), h_{n}(y)\right) \\
& C^{-1} \mathbf{d}\left(h_{n}^{-1}(x), h_{n}^{-1}(y)\right) \leq \mathbf{d}(x, y) \leq C \mathbf{d}\left(h_{n}^{-1}(x), h_{n}^{-1}(y)\right)
\end{aligned}
$$


for all $x, y \in \mathbf{S}^{3}, n=1,2, \ldots$ and a fixed positive constant $C$. Furthermore, the sequence $h_{n}$ converges to $h \in \operatorname{Aut}\left(\mathbf{S}^{3}\right)$ where

$$
\begin{aligned}
h(a) & =\mathrm{e}_{1}, \\
h(b) & =\mathrm{e}_{3}, \\
h(((\nu))) & =\mathrm{e}_{2}, \\
h(p) & =\mathrm{e}_{4}, \\
\mathbb{L}(h)(\nu) & =(0,1,0) .
\end{aligned}
$$

6.8. Normalization. We can conjugate by a sequence of $h_{n}$ of orientationpreserving bi-Lipschitz maps considered as an element of $\operatorname{Aut}\left(\mathbf{S}^{3}\right)$ so that $h_{n} g_{n} h_{n}^{-1}$ is of form

$$
\hat{g}_{n}:=\left[\begin{array}{cccc}
\lambda\left(g_{n}\right) & 0 & 0 & 0 \\
0 & 1 & 0 & \mu\left(g_{n}\right) \\
0 & 0 & \lambda\left(g_{n}\right)^{-1} & 0 \\
0 & 0 & 0 & 1
\end{array}\right]
$$

where $\lambda\left(g_{n}\right)>1$ and the orientation preserving $h_{n}$ is given by sending $\mathrm{e}_{1}$ to $a_{n}, \mathrm{e}_{2}$ to $\left(\left(\nu_{n}\right)\right), \mathrm{e}_{3}$ to $r_{n}$, and $\mathrm{e}_{4}$ to $p_{n}$. Also,

$$
g_{n}=h_{n}^{-1} \circ \hat{g}_{n} \circ h_{n} ;
$$

hence, $g_{n}$ acts as the standard form matrix in (23) does up to coordinatization by $h_{n}$.

The closed geodesic $c_{n} \subset \Sigma_{+}$corresponding to $g_{n}$ has length $l_{\mathbb{S}_{+}}\left(g_{n}\right)=$ $2 \log \left(\lambda\left(g_{n}\right)\right)$ for the largest eigenvalue $\lambda\left(g_{n}\right)$ of $g_{n}$. Recall that $\left\{g_{n}\right\}$ has a property (19):

Lemma 6.4. $\lambda\left(g_{n}\right) \longrightarrow+\infty, \quad \mu\left(g_{n}\right) \longrightarrow+\infty$, and $\frac{\mu\left(g_{n}\right)}{\lambda\left(g_{n}\right)} \longrightarrow 0$ as $i \longrightarrow \infty$.

Proof. Since $\left\{g_{n}\right\}$ restricts to a sequence of mutually distinct hyperbolic isometries in $\mathbb{S}_{+}$with sequences of fixed points $\left\{a_{n}\right\} \rightarrow a$ and $\left\{r_{n}\right\} \rightarrow b$ and $a \neq b$, it follows that $\left\{\lambda\left(g_{n}\right)\right\} \rightarrow \infty$ by the discreteness of $\mathbb{L}(\Gamma)$. By (13), $\mu\left(g_{n}\right) \rightarrow \infty$. By the main corollary of [46], $\mu\left(g_{n}\right) \leq C^{\prime} l_{\mathbb{S}_{+}}\left(g_{n}\right)$ and hence $\mu\left(g_{n}\right) \leq 2 C^{\prime}\left|\log \lambda\left(g_{n}\right)\right|$ hold for $C^{\prime}>0$; we obtain the final limit.

The set

$$
\hat{\Lambda}:=\mathscr{H}-(\mathrm{E} \cup \tilde{\Sigma})
$$

is compact. Moreover,

$$
\hat{\Lambda}=\bigcup_{z \in \Lambda} \mathrm{Cl}(\varepsilon(z))
$$

for the limit set $\Lambda$ of $\Gamma$. We recall from Lemma 3.4.

- the stable sphere $\mathbf{S}_{0}^{2}$ given by $x=0$, 
- the segment $\eta_{+}:=\overline{\mathrm{e}_{1} \mathrm{e}_{2} \mathrm{e}_{1-}}$,

- the segment $\eta_{-}:=\overline{\mathrm{e}_{3} \mathrm{e}_{2-} \mathrm{e}_{3-}}$.

Then:

- $h_{n}^{-1}\left(\eta_{-}\right)=\mathrm{Cl}\left(\varepsilon\left(r_{n}\right)\right)$ equals $\overline{r_{n}\left(\left(-\nu_{n}\right)\right) r_{n-}} \subset \hat{\Lambda}$ and

- $h_{n}^{-1}\left(\eta_{+}\right)=\mathrm{Cl}\left(\varepsilon\left(a_{n}\right)\right)$ equals $\overline{a_{n}\left(\left(\nu_{n}\right)\right) a_{n-}} \subset \hat{\Lambda}$.

6.9. The convergence of convex balls. Cover $K$ by compact convex sets and consider how their $g_{n}$-images transform. By $\$ 6.7$, we may assume by choosing a subsequence of $g_{n}$ satisfying

- $S_{n} \rightarrow S_{\infty}$ a great 2-dimensional sphere,

- $h_{n}^{-1}\left(\eta_{-}\right) \rightarrow \eta_{-}^{\infty}=\mathrm{Cl}(\varepsilon(b)) \subset \hat{\Lambda}$, and

- $h_{n}^{-1}\left(\eta_{+}\right) \rightarrow \eta_{+}^{\infty}=\mathrm{Cl}(\varepsilon(a)) \subset \hat{\Lambda}$.

Here

- $\eta_{+}^{\infty}$ is a segment $\overline{a((\nu)) a_{-}} \subset \hat{\Lambda}$ and

- $\eta_{-}^{\infty}$ is a segment $\overline{b((-\nu)) b_{-}} \subset \hat{\Lambda}$.

They are all tangent to $\partial \mathbb{S}_{+}$and

$$
K \subset \mathrm{E} \cup \tilde{\Sigma}=\mathscr{H}-\hat{\Lambda} \quad \subset \quad \mathscr{H}-\bigcup_{n=1}^{\infty} h_{n}^{-1}\left(\eta_{-}\right) .
$$

Let $\mathscr{H}_{a}$ and $\mathscr{H}_{a_{-}}$denote the respective components of $\mathscr{H}-S_{\infty}$ containing $a$ or $a_{-}$. We cover $K$ by balls $C_{j}(j \in \mathfrak{J})$ bounded away from $S_{\infty}$ and by balls $B_{l}(l \in \mathfrak{L})$ meeting $S_{\infty}$ precisely at the centers.

First, we cover $K$ as follows:

- $K_{\infty}:=K \cap S_{\infty}$. is a compact subset of an open 3-dimensional hemisphere in $S_{\infty}-\eta_{-}^{\infty}$. Cover $K_{\infty}$ by the interiors $\widetilde{B_{l}^{o}}$ of finitely many balls

$$
\widetilde{B_{l}} \subset \mathrm{E} \cup \tilde{\Sigma}
$$

with centers in $S_{\infty}$ for $l \in \mathfrak{L}$ of small d-radius $\epsilon_{0}>0$ in $\mathscr{H}-\hat{\Lambda}$. (We assume that balls $\hat{B}_{l}$ of $\mathbf{d}$-radius $2 \epsilon_{0}$ with the same centers remain in $\mathscr{H}-\hat{\Lambda}$.)

- Choose a foliation in $\mathscr{H}$ by perpendicular segments to $S_{\infty}$. We divide the ball $\widetilde{B_{l}}$ into the components $B_{l}^{+}, B_{l}^{-}$of $\widetilde{B_{l}}-S_{\infty}$ for each $l \in \mathfrak{L}$. Using isotopies $i_{+, l}$ and $i_{-, l}$ preserving the foliation so that

- the images

$$
\begin{aligned}
& C_{l}^{+} \subset \mathscr{H}_{a} \text { for } C_{l}^{+}:=i_{+, l}\left(\mathrm{Cl}\left(B_{l}^{+}\right)\right), \\
& C_{l}^{-} \subset \mathscr{H}_{a_{-}} \text {for } C_{l}^{-}:=i_{-, l}\left(\mathrm{Cl}\left(B_{l}^{-}\right)\right),
\end{aligned}
$$

are compact and convex, and 
- the dumbbell Join $\left(C_{l}^{+}, C_{l}^{-}\right)$remains in $\hat{B}_{l} \subset \mathrm{E} \cup \tilde{\Sigma}$ by displacing exclusively in the $2 \epsilon_{0}$-balls.

- Furthermore

$$
\begin{aligned}
K_{\infty} & \subset \bigcup_{l \in \mathfrak{L}} \operatorname{Join}\left(C_{l}^{+}, C_{l}^{-}\right)^{o} \\
& \subset \mathrm{E} \cup \tilde{\Sigma}
\end{aligned}
$$

and for all $l \in \mathfrak{L}$,

$$
\begin{aligned}
& \mathbf{d}\left(C_{l}^{+}, S_{\infty}\right)>\delta_{0}, \\
& \mathbf{d}\left(C_{l}^{-}, S_{\infty}\right)>\delta_{0}
\end{aligned}
$$

for some $\delta_{0}>0$.

- We obtain a compact subset

$$
\begin{aligned}
K^{\prime}:= & K-\bigcup_{l \in \mathfrak{L}} \operatorname{Join}\left(C_{l}^{+}, C_{l}^{-}\right)^{o} \\
\subset & (\mathrm{E} \cup \tilde{\Sigma})-S_{\infty} \\
& =\mathscr{H}-\left(\hat{\Lambda} \cup S_{\infty}\right) .
\end{aligned}
$$

Let $\epsilon_{K^{\prime}}:=\mathbf{d}\left(S_{\infty}, K^{\prime}\right)$. Find a finite set of $\mathbf{d}$-balls $B_{j}$, where $j \in \mathfrak{J}$, such that:

$$
\begin{aligned}
& -K^{\prime} \subset \bigcup_{j \in \mathfrak{J}} B_{j} ; \\
& -B_{j} \text { has radius } \frac{\epsilon_{K^{\prime}}}{2} ;
\end{aligned}
$$

$$
\mathbf{d}\left(B_{j}, S_{\infty}\right) \geq \frac{\epsilon_{K^{\prime}}}{2}
$$

Divide $\mathfrak{J}$ into $\mathfrak{J}^{+}$and $\mathfrak{J}^{-}$so that $j \in \mathfrak{J}^{+}$if and only if $B_{j} \subset \mathscr{H}_{a}$, and $j \in \mathfrak{J}^{-}$if and only if $B_{j} \subset \mathscr{H}_{a_{-}}$.

\subsection{The balls which are bounded away from $S_{\infty}$.}

Lemma 6.5. Let $g_{n}$ be a sequence as above and let

$$
\mathscr{B}:=\bigcup_{j \in \mathfrak{J}^{+}} B_{j} \cup \bigcup_{j \in \mathfrak{J}^{-}} B_{j} \cup \bigcup_{l \in \mathfrak{L}}\left(C_{l}^{+} \cup C_{l}^{-}\right) \text {. }
$$

Then as $n \longrightarrow \infty$, the sequence of sets $\left\{g_{n}(\mathscr{B})\right\}$ converges to $\{a,-a\}$, or to one of its subsets $\{a\}$ or $\{-a\}$.

Proof. For sufficiently small $\delta>0$, there exists $N_{0}$ such that for $n>N_{0}$

$$
\mathbf{d}\left(S_{n}, \mathscr{B}\right)>\delta
$$

by (27) and (28) since $S_{n} \rightarrow S_{\infty}$. 
Since $h_{n}\left(S_{n}\right)=\mathbf{S}_{0}^{2}, 22$ implies

$$
\mathbf{d}\left(\mathbf{S}_{0}^{2}, h_{n}(\mathscr{B})\right)>C^{-1} \delta
$$

for $n>N_{0}$. We decompose $\mathscr{B}=\mathscr{B}^{+} \cup \mathscr{B}^{-}$where

$$
\mathscr{B}^{+}:=\bigcup_{j \in \mathfrak{J}^{+}} B_{j} \cup \bigcup_{l \in \mathfrak{L}} C_{l}^{+} \text {and } \mathscr{B}^{-}:=\bigcup_{j \in \mathfrak{J}^{-}} B_{j} \cup \bigcup_{l \in \mathfrak{L}} C_{l}^{-} .
$$

Now act by $\hat{g}_{n}$ and apply Lemma 3.4 (a): For any $\epsilon^{\prime \prime}>0$, there exists $N_{1}$ so that for $n>N_{1}$,

$$
\hat{g}_{n} h_{n}\left(\mathscr{B}^{+}\right) \subset N_{\epsilon^{\prime \prime}}\left(\mathrm{e}_{1}\right), \hat{g}_{n} h_{n}\left(\mathscr{B}^{-}\right) \subset N_{\epsilon^{\prime \prime}}\left(\mathrm{e}_{1-}\right)
$$

hold.

Hence, for every $\epsilon^{\prime \prime}>0$, there exists $N_{1}>N_{0}$ so that for $n>N_{1}$, the sequence of the images of these sets under $g_{n}$ go into the $\epsilon^{\prime \prime}-\mathbf{d}$ neighborhoods of $a_{n}$ or $a_{n-}$ by (22). Since $a_{n} \rightarrow a$ and $a_{n-} \rightarrow a_{-}$,

$$
\mathbf{d}^{H}\left(\left\{a, a_{-}\right\}, g_{n}(\mathscr{B})\right) \rightarrow 0
$$

when $\mathscr{B}^{+}$and $\mathscr{B}^{-}$are both nonempty. The other cases are similar.

If $K_{\infty}=\emptyset$, then the reader can proceed to $\S 6.12$. Thus assume $K_{\infty} \neq \emptyset$ from now on.

6.11. The balls meeting $S_{\infty}$. We show that the dumbbells all converge to the semicircle outside of $\mathrm{E} \cup \tilde{\Sigma}$.

Lemma 6.6. For each $l \in \mathcal{L}$, the images $g_{n}\left(\right.$ Join $\left.\left(C_{l}^{+}, C_{l}^{-}\right)\right)$converge to the great semicircle $\eta_{+}^{\infty}=\overline{a((\nu)) a_{-}}$.

Proof. Since $\bigcup_{l \in \mathcal{I}} \hat{B}_{l}$ and $\hat{\Lambda}$ are disjoint compact subsets of $\mathscr{H}$,

$$
\mathrm{d}\left(\bigcup_{l \in \mathcal{L}} \operatorname{Join}\left(C_{l}^{+}, C_{l}^{-}\right), \hat{\Lambda}\right)>\delta^{\prime}
$$

for some $\delta^{\prime}>0$. We define

$$
C_{l, n}:=S_{n} \cap \operatorname{Join}\left(C_{l}^{+}, C_{l}^{-}\right)
$$

for $l \in \mathcal{L}$. Since $\mathrm{Cl}\left(\varepsilon\left(r_{n}\right)\right)$ lies in the compact set $\hat{\Lambda}$,

$$
\mathbf{d}\left(C_{l, n}, \mathrm{Cl}\left(\varepsilon\left(r_{n}\right)\right)\right)>\delta^{\prime}
$$

for a fixed $\delta^{\prime}>0$. Thus by 22,

$$
\mathbf{d}\left(h_{n}\left(C_{l, n}\right), \eta_{-}\right)>C^{-1} \delta^{\prime}
$$

for all $l \in \mathcal{L}$. By Lemma 3.4, for every $\epsilon>0$, there exists $N_{2}$ (independent of $l$ ) such that

$$
\hat{g}_{n} \circ h_{n}\left(C_{l, n}\right) \subset N_{\epsilon}\left(\mathrm{e}_{2}\right)
$$


for $n>N_{2}$. By 22,

$$
g_{n}\left(C_{l, n}\right) \subset N_{C \epsilon}\left(\left(\left(\nu_{n}\right)\right)\right)
$$

for $n>N_{2}$. Therefore (24) implies

$$
\lim _{n \rightarrow \infty} g_{n}\left(C_{l, n}\right)=((\nu)) .
$$

Lemmas 2.2 and 6.5 imply

$$
\lim _{n \rightarrow \infty} g_{n}\left(\operatorname{Join}\left(C_{l}^{+}, C_{l, n}\right)\right)=\overline{a((\nu))}
$$

since

$$
g_{n}\left(\operatorname{Join}\left(C_{l}^{+}, C_{l, n}\right)\right)=\operatorname{Join}\left(g_{n}\left(C_{l}^{+}\right), g_{n}\left(C_{l, n}\right)\right) .
$$

Similarly,

$$
\lim _{n \rightarrow \infty} g_{n}\left(\operatorname{Join}\left(C_{l}^{-}, C_{l, n}\right)\right)=\overline{((\nu)) a_{-}} .
$$

Since

$$
\operatorname{Join}\left(C_{l}^{+}, C_{l, n}\right) \cup \operatorname{Join}\left(C_{l}^{-}, C_{l, n}\right)=\operatorname{Join}\left(C_{l}^{+}, C_{l}^{-}\right),
$$

and

$$
\overline{a((\nu))} \cup \overline{((\nu)) a_{-}}=\eta_{+}^{\infty},
$$

facts in $\$ 2.4$ imply

$$
\lim _{n \rightarrow \infty} g_{n}\left(\operatorname{Join}\left(C_{l}^{+}, C_{l}^{-}\right)\right)=\eta_{+}^{\infty}
$$

for each $l \in \mathcal{L}$.

6.12. Conclusion of the proof when attractors and repellers differ. By our assumption of equation (18), $g_{n}(K) \cap K \neq \emptyset$ for all $n$. There has to be some fixed pair of balls $B$ and $B^{\prime}$ of one of the types:

- $B_{j}$ where $j \in \mathfrak{J}$

- Join $\left(C_{l}^{+}, C_{l}^{-}\right)$for $l \in \mathcal{L}$

so that $g_{n}(B) \cap B^{\prime} \neq \emptyset$ for infinitely many $n$. However, Lemma 6.5 and (29) imply that $g_{n}(B)$ converges to one of: $\eta_{+}^{\infty},\{a\},\left\{a_{-}\right\} \subset \mathbf{S}_{\infty}^{2}$. Thus, for each $\epsilon>0$ and every ball $B$ in the collection indexed by $\mathfrak{L} \cup \mathfrak{J}$, for sufficiently large $n$,

$$
N_{\epsilon}\left(\eta_{+}^{\infty}\right) \supset g_{n}(B)
$$

by definition of the geometric convergence. However, since the compact sets $\eta^{\infty}$ and $B^{\prime}$ are disjoint, $N_{\epsilon}\left(\eta_{+}^{\infty}\right) \cap B^{\prime}=\emptyset$ for any ball $B^{\prime}$ indexed by $\mathfrak{L} \cup \mathfrak{J}$ for sufficiently small $\epsilon>0$, obtaining a contradiction. 
6.13. Conclusion of the proof when attractors and repellers coincide. Now we consider the $a=r$ case. Since $\Gamma$ is a nonelementary Fuchsian group, choose an element $\gamma_{0} \in \Gamma$ so that

$$
\gamma_{0}(a) \neq a \text {. }
$$

Consider the sequence $\left\{\gamma_{0} g_{i}\right\}$. For each $\epsilon>0$ and a precompact neighborhood $U$ in $\partial \mathbb{S}_{+}-\{a\}$, there exists an integer $I_{0}$ such that

$$
\gamma_{0} g_{n}(U) \subset N_{\epsilon}\left(\gamma_{0}(a)\right) \text { for } n>I_{0} .
$$

Now consider the sequence $g_{n}^{-1} \gamma_{0}^{-1}$. Let $V$ be a precompact neighborhood in $\partial \mathbb{S}_{+}-\left\{\gamma_{0}(a)\right\}$. Since $\gamma_{0}^{-1}(V)$ is a precompact neighborhood in $\partial \mathbb{S}_{+}-\{a\}$ and for arbitrary $\epsilon>0$, there exists $I_{0}$ such that

$$
g_{n}^{-1}\left(\gamma_{0}^{-1}(V)\right) \subset N_{\epsilon}(a) \text { for } n>I_{0} .
$$

Thus, $\gamma_{0}(a)$ is the attractor point and $a$ is the repeller point of the sequence $\left\{\gamma_{0} g_{n}\right\}$. (See $\S 4.3$.)

Since

$$
\gamma_{0} g_{n}\left(K \cup \gamma_{0}(K)\right) \cap\left(K \cup \gamma_{0}(K)\right) \neq \emptyset
$$

for infinitely many $n$, we are reduced to the case where $a \neq r$ by replacing $g_{n}$ with $\gamma_{0} g_{n}$ and $K$ with another compact set

$$
K \cup \gamma_{0}(K) \subset \mathrm{E} \cup \tilde{\Sigma} .
$$

Again, we obtain contradiction.

\section{TAMENESS.}

7.1. The compactification. Using the above notations, we note that $\tilde{\Sigma} / \Gamma$ is a closed surface of genus $g$ and forms the boundary of the 3 manifold $M:=(\mathrm{E} \cup \tilde{\Sigma}) / \Gamma$ by Proposition 6.2 provided $\mathbb{L}(\Gamma) \subset \mathrm{SO}(2,1)^{\circ}$. Therefore, $M$ is a 3 -manifold in general since $\Gamma$ has an index $\leq 2$ subgroup $\Gamma^{\prime}$ with the property $\mathbb{L}\left(\Gamma^{\prime}\right) \subset \mathrm{SO}(2,1)^{o}$ and the fact that $\Gamma$ acts freely on $\mathrm{E} \cup \tilde{\Sigma}$.

We now show that $M$ is compact. The key idea is to isotopy some spheres into $\mathrm{E}$ bounding compact 3-balls. We first assume that $\mathbb{L}(\Gamma) \subset$ $\mathrm{SO}(2,1)^{o}$ so that $\Gamma$ acts on $\mathbb{S}_{+}$honestly.

Proposition 7.1. Each simple closed curve $\gamma$ in $\tilde{\Sigma}$ bounds a simple disk in $\mathrm{E} \cup \tilde{\Sigma}$. Let $c$ be a simple closed curve in $\Sigma$ that is homotopically trivial in $M$. Then $c$ bounds an embedded disk in $M=(\mathrm{E} \cup \tilde{\Sigma}) / \Gamma$.

Proof. This is just Dehn's lemma.

We can find a collection of disjoint simple curves $\gamma_{i}, i \in \mathcal{J}^{\prime}$, on $\tilde{\Sigma}$ for an index set $\mathcal{J}^{\prime}$ so that the following hold:

- $\bigcup_{i \in \mathcal{J}^{\prime}} \gamma_{i}$ is invariant under $\Gamma$. 


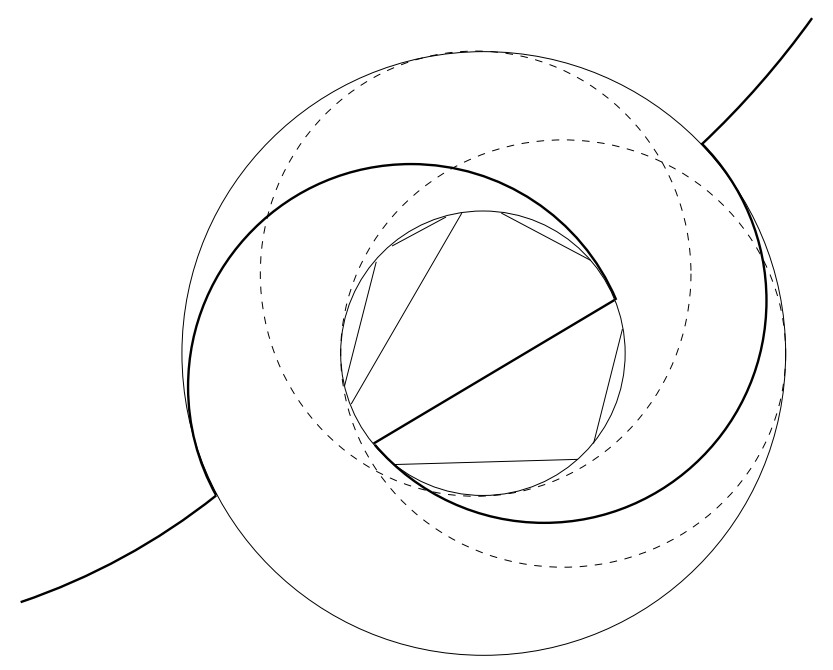

Figure 6 . The arcs in $\mathbb{S}_{+}$and an example of $\hat{\gamma}_{i}$ in the bold arcs.

- $\bigcup_{i \in \mathcal{J}^{\prime}} \gamma_{i}$ cuts $\tilde{\Sigma}$ into a union of open pair-of-pants $P_{k}, k \in K$, for an index set $K$. The closure of each $P_{k}$ is a closed pair-of-pants.

- $\left\{P_{k}\right\}_{k \in K}$ is a $\Gamma$-invariant set.

- Under the covering map $\pi: \tilde{\Sigma} \rightarrow \tilde{\Sigma} / \Gamma$, each $\gamma_{i}$ for $i \in \mathcal{J}^{\prime}$ maps to a simple closed curve in a one-to-one manner and each $P_{k}$ for $k \in K$ embeds onto an open pair-of-pants.

This is achieved by finding arcs in $\mathbb{S}_{+} / \Gamma$ cutting it into disks. Recall that $\mathbb{S}_{+} / \Gamma$ is an open surface compactified to the compact surface $\Sigma_{+}^{\prime}$ with boundary. We obtain a system of geodesic segments $\hat{\alpha}_{i}, i \in I$ for a finite set $I$, in $\Sigma_{+}^{\prime}$ cutting $\Sigma_{+}^{\prime}$ into a union of disks. Each disk is bounded by six arcs, alternating triple of which are arcs coming from $\partial \Sigma_{+}^{\prime}$ and the other alternating triples are geodesic segments ending $\partial \Sigma_{+}^{\prime}$. We can assume that each $\hat{\alpha}_{i}$ is embedded in $\tilde{\Sigma}_{+} / \Gamma$ cutting it into finitely many hexagonal disks so that each ideal boundary component of $\tilde{\Sigma}_{+} / \Gamma$ meets at least two arcs of form $\hat{\alpha}_{i}$.

This gives a system of geodesic arcs $\alpha_{j}, j \in \mathcal{J}^{\prime}$, in $\mathbb{S}_{+}$for some infinite index set $\mathcal{J}^{\prime}$ which decomposes $\tilde{\Sigma}_{+}$into hexagonal disks and forms a $\Gamma$-invariant set. We define $\alpha_{j-}:=\mathscr{A}\left(\alpha_{j}\right)$ in $\mathrm{Cl}\left(\mathbb{S}_{-}\right)$for $j \in \mathcal{J}^{\prime}$. We connect each endpoint $\partial_{1}\left(\alpha_{j}\right)$ and $\partial_{2}\left(\alpha_{j}\right)$ of $\alpha_{j}$ with its antipodal endpoint of $\alpha_{j-}$ by arcs of form $\varepsilon(x)$.

$$
\gamma_{j}:=\alpha_{j} \cup \alpha_{j-} \cup \varepsilon\left(\partial_{1}\left(\alpha_{j}\right)\right) \cup \varepsilon\left(\partial_{2}\left(\alpha_{j}\right)\right), j \in \mathcal{J}^{\prime} .
$$

(These form crooked circles.) We do this for each arc and obtain the above system of simple closed curves. By construction, $\left\{\gamma_{i}\right\}_{i \in \mathcal{J}^{\prime}}$ maps to a system of disjointly embedded curves $\hat{\gamma}_{1}, \ldots, \hat{\gamma}_{3 \mathbf{g}-3}$ in $\Sigma$, and each of them has a simple closed lift in $\tilde{\Sigma}$ and hence has a trivial holonomy. 
(See Figure 6.) Also, the collection $\hat{\gamma}_{1}, \ldots, \hat{\gamma}_{3 \mathrm{~g}-3}$ decompose the closed surface $\tilde{\Sigma} / \Gamma$ of genus $\mathbf{g}$ for some $\mathbf{g} \geq 2$ into $2 \mathbf{g}-2$ pairs of pants $P_{1}^{\prime}, \ldots, P_{2 \mathbf{g}-2}^{\prime}$. (Here $\mathbf{g}=2 \tilde{\mathbf{g}}+\mathrm{b}-1$ for the genus $\tilde{\mathbf{g}}$ of $\mathbb{S}_{+} / \Gamma$ and the number $\mathrm{b}$ of ideal boundary components.)

By trivial holonomy and Dehn's lemma, each $\hat{\gamma}_{i}$ bounds a disk $D_{i}$ in $M$. By the 3-manifold topology of disk exchanges, we can choose $D_{1}, \ldots, D_{3 \mathrm{~g}-3}$ to be mutually disjoint. (See [49].)

Each pair of pants $P_{j}^{\prime}$ and union with three adjacent ones in the collection $D_{1}, \ldots, D_{3 \mathbf{g}-3}$ is homeomorphic to a 2 -sphere in $M$. We can lift the sphere into $E \cup \tilde{\Sigma}$ and being a sphere which can be pushed inside the cell $\mathrm{E}$, it bounds a compact 3-ball $B$ in $\mathrm{E} \cup \tilde{\Sigma}$. The image of $B$ is a compact 3-ball in $M$ as well since $\pi \mid \partial B$ is an embedding to a 2-sphere and the image of $B^{o}$ is disjoint from all other disks and $\pi$ is a covering map. Therefore, the Cauchy completion of each component of $M-D_{1}-\cdots-D_{3 \mathbf{g}-3}$ is homeomorphic to a compact 3-ball in $M$.

Since $M$ is a union of the closure of the components that are 3-balls identified with one another in disjoint disks, $M$ is a compact 3-manifold. This implies also that $M$ is homeomorphic to a solid handlebody of genus g; that is, our manifold $(E \cup \tilde{\Sigma}) / \Gamma$ is a compact 3-manifold and so its interior $E / \Gamma$ is tame. This completes the proof of Theorem 1.2 provided $\mathbb{L}(\Gamma) \subset \mathrm{SO}(2,1)^{\circ}$. (Also, it follows that the rank of $\Gamma$ equals g.)

In the general case when $\mathbb{L}(\Gamma)$ is not a subgroup of $\mathrm{SO}(2,1)^{\circ}$, the above work applies to $(E \cup \tilde{\Sigma}) / \Gamma^{\prime}$ double-covering $(E \cup \tilde{\Sigma}) / \Gamma$. By Theorem 5.2 of Chapter 5 of [49], $M$ is homeomorphic to a handlebody since $\Gamma$ is a free group and $(E \cup \tilde{\Sigma}) / \Gamma$ is an aspherical compact 3-manifold.

We conclude:

Theorem 7.2 (Compactification). Let $\mathrm{E} / \Gamma$ be a Margulis spacetime and $\Gamma$ is a Lorentzian isometry group without parabolics. Suppose that the Margulis invariants of elements of $\Gamma-\{\mathrm{I}\}$ are all positive. Choose $\tilde{\Sigma}$ by (11). Then $(\mathrm{E} \cup \tilde{\Sigma}) / \Gamma$ is homeomorphic to a compact handlebody.

If we change the orientation or make the Margulis invariants to be all negative, then the conclusion holds for $(E \cup \mathcal{A}(\tilde{\Sigma})) / \Gamma$. If we do both, then the conclusion holds for $(E \cup \tilde{\Sigma}) / \Gamma$.

7.2. The almost-crooked-plane decomposition. A crooked plane is a closed disk embedded in $\mathrm{E}$ constructed as follows: Take two null vectors $\mathrm{v}_{1}$ and $\mathrm{v}_{2}$ and respective parallel lines $L_{1}$ and $L_{2}$ meeting at a point $x$. Then they are on a timelike plane $P$. Take all timelike lines on $P$ from $x$ and take their union. It is a union of two cones $C_{1}$ and $C_{2}$ with vertex at $x$. We obtain two null half-planes $\mathscr{W}\left(\mathrm{v}_{1}\right)$ and $\mathscr{W}\left(\mathrm{v}_{2}\right)$ 
containing $x$. A crooked plane is the union $C_{1} \cup C_{2} \cup \mathscr{W}\left(\mathrm{v}_{1}\right) \cup \mathscr{W}\left(\mathrm{v}_{2}\right)$. (See [30] for details.)

A disk in $\mathrm{E}$ is an almost crooked plane if it agrees with a crooked plane in the complement of its compact subset. Also, its immersed or embedded image is said to be an almost crooked plane as well.

Proposition 7.3. Let $\tilde{\Sigma}$ and $\Gamma$ be as above. The Margulis spacetime $\mathrm{E} / \Gamma$ without cusp has a system of disks with boundary in $(\mathrm{E} \cup \tilde{\Sigma}) / \Gamma$ so that the closures of components of the complement are compact 3-balls and the disks are almost crooked planes.

Proof. First, assume $\mathbb{L}(\Gamma) \subset \mathrm{SO}(2,1)^{\circ}$. Each of our disks has the boundary of a crooked plane. In Dehn's lemma, one can arbitrarily assign the tubular neighborhood of the boundary of each disk as long as it is transversal to $\tilde{\Sigma} / \Gamma$. Thus, following the above discussions, we obtain the proof in this case.

Suppose that $\mathbb{L}(\Gamma)$ is not in $\mathrm{SO}(2,1)^{\circ}$. Then we take a quotient $\Sigma^{\prime}:=$ $\mathbb{S}_{+} / \mathbb{L}^{\prime}(\Gamma)$ given by the projective action. The nonorientable $\Sigma^{\prime}$ admits a decomposition into hexagonal disks as above. We obtain the induced decomposition on $\mathbb{S}_{+} \cup \mathbb{S}_{-}$and we extend the above construction to this situation and obtain the crooked circles and crooked planes.

As above $(\mathrm{E} \cup \tilde{\Sigma}) / \Gamma^{\prime}$ double-covers $(\mathrm{E} \cup \tilde{\Sigma}) / \Gamma$ with the deck transformation group generated by a projective automorphism $\phi$ of order two. We choose a collection for the quotient $\mathbb{S}_{+} / \mathbb{L}^{\prime}(\Gamma)$ as in the beginning of the proof of Proposition 7.1. Then $\phi$ acts on the system of circles by construction. We can modify each almost crooked plane $D$ not changing a neighborhood of $\partial D$ in $D$ so that $D \cap \phi(D)=\emptyset$ using the proof of Theorem 3 of [48]. Hence, we obtain a collection of $\phi$-equivariant almost crooked planes that cuts $M$ into 3-balls.

Corollary 7.4. A Margulis spacetime without cusp has a finite sided fundamental polyhedron in $\mathrm{E}$.

Proof. We take a union of finitely many of the 3-balls obtained by cutting along the disks in $\mathrm{E}$.

\section{REFERENCES}

[1] H. Abels, Properly discontinuous groups of affine transformations: a survey, Geom. Dedicata 87 (2001), 309-333.

[2] H. Abels, G.A. Margulis, and G.A. Soifer, Properly discontinuous groups of affine transformations with orthogonal linear part, C. R. Acad. Sci. Paris Sér. I Math. 324 (1997), 253-258.

[3] I. Agol, Tameness of hyperbolic 3-manifolds, math.GT/0405568

[4] J. Anderson, P. Bonfert-Taylor, and E. Taylor, Convergence groups, Hausdorff dimension, and a theorem of Sullivan and Tukia, Geom. Dedicata 103 (2004), $51-67$. 
[5] A. Beardon, Geometry of discrete groups, Graduate Text in Mathematics Vol. 91 Springer New York, 1995.

[6] Y. Benoist, Convexes divisible, C.R. Acad. Sci. 332 (2003), 387-390.

[7] J.-P. Benzécri, Sur les variétés localement affines et localement projectives, Bull. Soc. Math. France 88 (1960), 229-332.

[8] F. Bonahon, Bouts des variétés hyperboliques de dimension 3, Ann. of Math. (2) 124 (1986), 71-158.

[9] B. Bowditch, Notes on tameness, Enseign. Math. (2) 56 (2010), 229-285.

[10] W. Browder, J. Levine, and G. Livesay, Finding a boundary for an open manifold, Amer. J. Math. 87 (1965), 1017-1028.

[11] H. Busemann and P. Kelly, Projective geometry and projective metrics, Academic Press Inc., New York, N. Y., 1953. viii+332 pp.

[12] D. Calegari and D. Gabai, Shrinkwrapping and the taming of hyperbolic 3manifolds, J. Amer. Math. Soc. 19 (2006), 385-446.

[13] R. Canary and Y. N. Minsky, On limits of tame hyperbolic 3-manifolds, J. Differential Geom. 43 (1996), 1-41.

[14] V. Charette and T. Drumm, Strong marked isospectrality of affine Lorentzian groups, J. Differential Geom. 66 (2004), 437-452.

[15] V. Charette, T. Drumm, and W. Goldman, Affine deformations of the threeholed sphere, Geom. Topol. 14 (2010), 1355-1382.

[16] V. Charette, T. Drumm, and W. Goldman, Finite-sided deformation spaces of complete affine 3-manifolds, Journal of Topology 7 (2014), 225-246.

[17] V. Charette, T. Drumm, and W. Goldman, Proper affine deformations of twogenerator Fuchsian groups, Transformation Groups 21 (2016) no. 4, 953-1002.

[18] V. Charette, W. Goldman, and C. Jones, Recurrent geodesics in flat Lorentzian manifolds, Canad. Math. Bull. 47 (2004), 332-342.

[19] S. Choi, http://mathsci.kaist.ac.kr/ schoi/action2.nb

[20] S. Choi, Convex decompositions of real projective surfaces. I: $\pi$-annuli and convexity, J. Differential Geom. 40 (1994), 165-208.

[21] S. Choi, Convex decompositions of real projective surfaces. II: Admissible decompositions, J. Differential Geom. 40 (1994), 239-283.

[22] S. Choi, The Margulis Lemma and the thick and thin decomposition for convex real projective surfaces, Adv. Math. 122 (1996), 150-191.

[23] S. Choi, The convex and concave decomposition of manifolds with real projective structures, Mémoires de la Société mathématique de France, Nouvelle série, Numéro 78, 1999. 110 pp.

[24] S. Choi and W. Goldman, The classification of real projective structures on compact surfaces, Bull. Amer. Math. Soc. (N.S.) 34 (1997), 161-171.

[25] S. Choi, T. Drumm and W. Goldman, Tameness of Margulis space-times with parabolics, preprint, 2015.

[26] D. Cooper, D. Long, and M. Thistlethwaite, Computing varieties of representations of hyperbolic 3-manifolds into SL(4, R), Exp. Math. 15 (2006), 291-305.

[27] D. Cooper, D. Long, and M. Thistlethwaite, Flexing closed hyperbolic manifolds, Geom. Topol. 11 (2007), 2413-2440.

[28] J. Danciger, F. Guéritaud, and F. Kassel, Geometry and topology of complete Lorentz spacetimes of constant curvature, Ann. Sci. Éc. Norm. Supér. 49 (2016), 1-56.

[29] J. Danciger, F. Guéritaud, and F. Kassel, Margulis spacetime via the arc complex, Invent. Math. 204 (2016), 133-193. 
[30] T. Drumm, Fundamental polyhedra for Margulis space-times, Doctoral dissertation, University of Maryland (1990); Topology 31 (1992), 677-683.

[31] T. Drumm, Linear holonomy of Margulis space-times, J. Differential Geom. 38 (1993), 679-691.

[32] T. Drumm and W. Goldman, Crooked planes, Electronic Research Announcements of the Amer. Math. Soc. 1 (1995), 10-17.

[33] T. Drumm and W. Goldman, The geometry of crooked planes, Topology 38 (1999), 323-351.

[34] T. Drumm and W. Goldman, The isospectrality of flat Lorentz 3-manifolds, J. Differential Geom. 58 (2001), 457-465.

[35] P. Eberlein, Geodesic flows on negatively curved manifolds, I, Ann. of Math., 2nd Ser. 95 (1972), 492-510.

[36] L. Eisenhart, Non-Riemannian geometry, Colloquium Publication Vol. 8 Amer. Math. Soc. Providence 1927.

[37] D.B.A. Epstein and A. Marden, Convex hulls in hyperbolic space, a theorem of Sullivan, and measured pleated surfaces, Analytical and geometric aspects of hyperbolic space (Coventry/Durham, 1984), pp. 113-253, London Math. Soc. Lecture Note Ser. Vol. 111, Cambridge Univ. Press, Cambridge, 1987.

[38] C. Frances, The conformal boundary of Margulis space-times, C.R. Acad. Sci. Paris, Ser. 1336 (2003), 751-756.

[39] D. Fried and W. Goldman, Three-dimensional affine crystallographic groups, Adv. Math. 47 (1983), 1-49.

[40] D. Fried, W. Goldman, and M. Hirsch, Affine manifolds with nilpotent holonomy, Comment. Math. Helv. 56 (1981), 487-523.

[41] W. Goldman, Affine manifolds and projective geometry on surfaces, Senior Thesis, Princeton University, 1977.

[42] W. Goldman, Projective structures with Fuchsian holonomy, J. Differential Geom. 25 (1987), 297-326.

[43] W. Goldman, Convex real projective structures on compact surfaces, J. Differential Geom. 31 (1990), 791-845.

[44] W. Goldman, Projective geometry of manifolds, Lecture notes from a 1988 course, http://www2.math. umd.edu/ wmg/pgom.pdf 1988.

[45] W. Goldman and F. Labourie, Geodesics in Margulis space times, Ergod. Theory Dynam. Systems 32 (2012), 643-651.

[46] W. Goldman, F. Labourie, and G. Margulis, Proper affine actions and geodesic flows of hyperbolic surfaces, Ann. of Math. (2) $\mathbf{1 7 0}$ (2009), 1051-1083.

[47] W. Goldman, F. Labourie, G. Margulis, and Y. Minsky, Complete flat Lorentz 3-manifolds and laminations on hyperbolic surfaces, preprint, May 29, 2012

[48] C. McA. Gordon and R.A. Litherland, Incompressible surfaces in branched coverings, Smith Conjecture (eds. Morgan and Bass), Academic Press 1984, pp. $139-151$.

[49] J. Hempel, 3-manifolds, Princeton University Press, Princeton, 1976.

[50] S. Katok and B. Hasselblatt, Introduction to the modern theory of dynamical systems, Encyclopedia of mathematics and its applications vol. 54, Cambridge University Press, Cambridge 1995.

[51] N. Kuiper, On convex locally-projective spaces, Convegno Internazionale di Geometria Differenziale (Italia, 1953), pp. 200-213. Edizioni Cremonese, Roma, 1954. 
[52] A. Marden, The geometry of finitely generated Kleinian groups, Ann. of Math. (2) 99 (1974), 383-462.

[53] G.A. Margulis, Complete affine locally flat manifolds with a free fundamental group, Automorphic functions and number theory, II, Zap. Nauchn. Sem. Leningrad. Otdel. Mat. Inst. Steklov (LOMI) 134, 1984, pp. 190-205.

[54] G. Mess, Lorentz space-times of constant curvature, Geom. Dedicata 126 (2007), 3-45.

[55] R. Meyers, End reductions, fundamental groups, and covering spaces of irreducible open 3-manifolds, Geom. Topol. 9 (2005), 971-990.

[56] J. Munkres, Topology (2nd edition), Prentice Hall, 1999.

[57] T. Nagano and K. Yagi, The affine structures on the real two-torus: I, Osaka J. of Math. 11 (1974), 181-210.

[58] P. Scott, Compact submanifolds of 3-manifolds, J. Lond. Math. Soc. (2) 7 (1973), 246-250.

[59] P. Scott and T. Tucker, Some examples of exotic noncompact 3-manifolds, $Q$. J. Math. Ser. (2) 40 (1989), 481-499.

[60] L. Siebenmann, The obstruction to finding a boundary for an open manifold of dimension greater than five, Ph.D. thesis, Princeton University, 1965.

[61] J. Souto, A note on the tameness of hyperbolic 3-manifolds, Topology 44 (2005) 459-474.

[62] D. Sullivan and W. Thurston, Manifolds with canonical coordinate charts: some examples, Enseign. Math. (2) 29 (1983), 15-25.

[63] W. Thurston, Geometry and topology of 3-manifolds, Lecture notes. Princeton University, 1979 http://www.msri.org/publications/books/gt3m/.

[64] T. Tucker, Non-compact 3-manifolds and the missing-boundary problem, Topology 13 (1974), 267-273.

Department of Mathematical Sciences, KAist, Daejeon 34141, RePUBLIC OF KOREA,

E-mail address: schoi@math.kaist.ac.kr

Department of Mathematics, University of Maryland, College Park, MD 20742-4015, USA

E-mail address: wmg@math.umd.edu 